\title{
HOPF-GALOIS STRUCTURES OF SQUAREFREE DEGREE
}

\author{
ALI A. ALABDALI AND NIGEL P. BYOTT
}

\begin{abstract}
Let $n$ be a squarefree natural number, and let $G, \Gamma$ be two groups of order $n$. We determine the number of Hopf-Galois structures of type $G$ admitted by a Galois extension of fields with Galois group isomorphic to $\Gamma$. We give some examples, including a full treatment of the case where $n$ is the product of three primes.
\end{abstract}

\section{INTRODUCTION}

The notion of Hopf-Galois extension was introduced by Chase and Sweedler [CS69]. (We recall the definition in $\S 3$ below.) This was in part motivated by the study of inseparable extensions of fields, but is also of considerable interest for separable extensions. Indeed, Greither and Pareigis [GP87] showed that a given separable extension of fields $L / K$ may admit many Hopf-Galois structures, and that finding them can be reduced to a group-theoretic problem. If $H$ is a $K$-Hopf algebra giving a Hopf-Galois structure on $L / K$, then, extending scalars to the algebraic closure $K^{c}$ of $K$, we have an isomorphism of $K^{c}$-Hopf algebras $K^{c} \otimes_{K} H \cong K^{c}[G]$, where $K^{c}[G]$ is the group algebra of a group $G$ whose order coincides with the degree of $L / K$. The isomorphism type of $G$ is called the type of the Hopf-Galois structure. Given abstract groups $\Gamma$, $G$ of the same finite order $n$, we would like to determine the number $e(\Gamma, G)$ of Hopf-Galois structures of type $G$ on a Galois extension $L / K$ with Galois group $\operatorname{Gal}(L / K) \cong \Gamma$.

Since the work of Greither and Pareigis, there have been a number of papers enumerating Hopf-Galois structures on Galois extensions of various kinds: see for example [Byo04, CC99, Chi03, CRV18, Koh98, Koh16, NZ19]. In [AB18], we considered cyclic extensions of squarefree degree $n$, obtaining a simple formula for the number of Hopf-Galois

Date: April 14, 2020.

2010 Mathematics Subject Classification. 12F10, 16 T05.

Key words and phrases. Hopf-Galois structures; field extensions; groups of squarefree order.

The first-named author acknowledges support from The Higher Committee for Education Development in Iraq (PhD studentship D-11-1775). 
structures whose type is any given group of order $n$. The purpose of this paper is to extend the work in [AB18] by allowing an arbitrary Galois group. Thus we determine $e(\Gamma, G)$ for any two groups $\Gamma, G$ of squarefree order $n$. The answer, which we state in Theorem 2.2 below once we have developed the necessary notation, depends on an interplay of the structures of the two groups, and turns out to be considerably more complicated than when $\Gamma$ is cyclic.

Most of this paper will be taken up with the proof of Theorem 2.2, but in $\S 8$ we present a fairly elaborate example to indicate the complexities that can occur. Then, in $\S 9$, we consider various special cases of Theorem 2.2, some of which recover results already in the literature. In particular, we give in Theorem 9.6 a full treatment of the case where $n$ is the product of three primes. Some partial results for this case were given in [Chi03, Koh13, Koh16].

The problem of enumerating Hopf-Galois structures on Galois extensions of fields is closely related (but not equivalent) to the problem of enumerating certain algebraic structures called skew braces, which were introduced by Guarnieri and Vendramin [GV17] to study set-theoretical solutions of the quantum Yang-Baxter equation. Skew braces generalise the braces defined by Rump in [Rum07]. The connection between braces and Hopf-Galois structures was first observed by Bachiller [Bac16], and the relationship between the two enumeration problems was clarified in the appendix to [SV18]. In a separate paper $[\mathrm{AB}]$, we apply the results obtained here to enumerate skew braces of squarefree order.

Much of the content of this paper, along with that of [AB18], appears in the $\mathrm{PhD}$ thesis [Ala18] of the first-named author. We take the opportunity here to make some improvements in the exposition and to correct some minor errors in [Ala18].

\section{Groups of SQUAREFree order AND MAIN RESUlt}

A finite group in which all Sylow subgroups are cyclic is necessarily metabelian, so such groups may be classified, see e.g. [MM84]. This applies in particular to groups of squarefree order, and combining [MM84, Lemmas 3.5, 3.6] in this case, we obtain the following result (cf. [AB18, Lemma 3.2]).

Lemma 2.1. Let $n \geq 1$ be a squarefree integer. Then any group of order $n$ has the form

$$
G(d, e, k)=\left\langle\sigma, \tau: \sigma^{e}=\tau^{d}=1_{G}, \tau \sigma \tau^{-1}=\sigma^{k}\right\rangle
$$


where $n=d e, \operatorname{gcd}(d, e)=1$ and $\operatorname{ord}_{e}(k)=d$. Conversely, any choice of $d$, e and $k$ satisfying these conditions gives a group $G(d, e, k)$ of order $n$. Moreover, two such groups $G(d, e, k)$ and $G\left(d^{\prime}, e^{\prime}, k^{\prime}\right)$ are isomorphic if and only if $d=d^{\prime}, e=e^{\prime}$, and $k, k^{\prime}$ generate the same cyclic subgroup of $\mathbb{Z}_{e}^{\times}$.

Here, for any natural numbers $a, m$ with $\operatorname{gcd}(a . m)=1$, we write $\operatorname{ord}_{m}(a)$ for the order of $a$ in the group $\mathbb{Z}_{m}^{\times}$of units in the ring $\mathbb{Z}_{m}$ of integers modulo $m$.

We now fix a squarefree number $n$ and a group $G=G(d, e, k)$ of order $n$, as in Lemma 2.1. Let

$$
z=\operatorname{gcd}(k-1, e), \quad g=e / z
$$

and for each prime $q \mid e$, let $r_{q}=\operatorname{ord}_{q}(k)$. In general, $d, g$ and $z$ do not determine the $r_{q}$. Moreover, $d, g, z$ and the $r_{q}$ do not determine the isomorphism type of $G$. This is illustrated by the examples in $\S 8$.

Next let $\Gamma$ be another group of order $n$. As far as possible, we will use corresponding Greek and Roman letters for quantities associated with $\Gamma$ and $G$. We will however continue to denote the generators of $G$ as $\sigma, \tau$ (and correspondingly write the generators of $\Gamma$ as $s, t$ ). We choose this somewhat awkward notation for the sake of consistency with [AB18]. Thus we write

$$
\Gamma=G(\delta, \epsilon, \kappa)=\left\langle s, t: s^{\epsilon}=t^{\delta}=1, t s t^{-1}=s^{\kappa}\right\rangle,
$$

with $\delta \epsilon=n$ and $\operatorname{ord}_{\epsilon} \kappa=\delta$, and we set

$$
\zeta=\operatorname{gcd}(\kappa-1, \epsilon), \quad \gamma=\epsilon / \zeta, \quad \rho_{q}=\operatorname{ord}_{q}(\kappa) \text { for primes } q \mid \epsilon .
$$

We consider the set

$$
\mathcal{K}=\left\{\kappa^{r}: r \in \mathbb{Z}_{\delta}^{\times}\right\} .
$$

By Lemma 2.1, we may replace $\kappa$ in (2.2) by any element of $\mathcal{K}$ without changing the isomorphism type of $\Gamma$. The group $\mathbb{Z}_{\delta}^{\times}$acts regularly on $\mathcal{K}$ by exponentiation, so its subgroup

$$
\Delta:=\left\{m \in \mathbb{Z}_{\delta}^{\times}: m \equiv 1 \quad(\bmod \operatorname{gcd}(\delta, d))\right\}
$$

acts without fixed points on $\mathcal{K}$. The index of $\Delta$ in $\mathbb{Z}_{\delta}^{\times}$is

$$
w=\varphi(\operatorname{gcd}(\delta, d)),
$$

where $\varphi$ is the Euler totient function. Let $\kappa_{1}, \ldots, \kappa_{w}$ be a system of orbit representatives of $\Delta$ on $\mathcal{K}$.

We now define two sets of primes, depending on both $G$ and $\Gamma$ :

$$
\begin{aligned}
& S=\left\{\text { primes } q \mid \operatorname{gcd}(\gamma, g): \rho_{q}=r_{q}>2\right\} \\
& T=\left\{\text { primes } q \mid \operatorname{gcd}(\gamma, g): \rho_{q}=r_{q}=2\right\}
\end{aligned}
$$


For $1 \leq h \leq w$, we also define

$$
S_{h}=\left\{q \in S: \kappa_{h} \equiv k \text { or } \kappa_{h} \equiv k^{-1} \quad(\bmod q)\right\} .
$$

We make some comments on the definitions of $\kappa_{h}$ and $S_{h}$ in Remarks $5.4,6.4$ and 6.5 below.

For a natural number $m$, let $\omega(m)$ denote the number of distinct prime factors of $m$. Thus, when $m$ is squarefree, $2^{\omega(m)}$ is the number of positive integer factors of $m$. Recall that $e(\Gamma, G)$ denotes the number of Hopf-Galois structures on a Galois extension $L / K$ with $\operatorname{Gal}(L / K) \cong \Gamma$. We warn the reader not to confuse this notation with the parameter $e$ in the group $G=G(d, e, k)$.

We can now state our main result.

Theorem 2.2. Let $G$ and $\Gamma$ be groups of squarefree order $n$. With the above notation, we have

$$
e(\Gamma, G)= \begin{cases}\frac{2^{\omega(g)} \varphi(d) \gamma}{w}\left(\prod_{q \in T} \frac{1}{q}\right) \sum_{h=1}^{w} \prod_{q \in S_{h}} \frac{q+1}{2 q} & \text { if } \gamma \mid e, \\ 0 & \text { if } \gamma \nmid e .\end{cases}
$$

Remark 2.3. In [AB18, Theorem 2], we gave a formula, as a sum over factorisations $n=d g z$ of $n$ into 3 factors, for the total number of Hopf-Galois structures on a cyclic extension of squarefree degree $n$. Attempts to give a similar formula when the Galois group is an arbitrary group $\Gamma$ of squarefree order $n$ lead to complicated multiple sums which do not appear to admit any significant simplification. We therefore do not give any results of this sort in this paper. For some partial results in this direction, see [Ala18, §5.5]

\section{Background on Hopf-Galois Structures}

Let $L / K$ be a finite extension of fields and let $H$ be a cocommutative $K$-Hopf algebra with a $K$-linear action on $L$. We say that $L / K$ is an $H$-Galois extension if the following conditions are satisfied:

(i) $h \cdot(x y)=\sum_{(h)}\left(h_{(1)} \cdot x\right)\left(h_{(2)} \cdot y\right)$ for all $h \in H$ and $x, y \in L$, where we use Sweedler's notation $h \mapsto \sum_{(h)} h_{(1)} \otimes h_{(2)}$ for the comultiplication of $H$;

(ii) $h \cdot 1=\varepsilon(h) 1$, where $\varepsilon: H \rightarrow K$ is the counit of $H$;

(iii) the $K$-linear map $\theta: L \otimes_{K} L \rightarrow \operatorname{Hom}_{K}(L, L)$ is bijective, where $\theta(x \otimes y)(h)=x(h \cdot y)$.

A Hopf-Galois structure on $L / K$ consists of a cocommutative $K$-Hopf algebra $H$, together with an action of $H$ on $L$ making $L / K$ into an $H$-Galois extension. 
Greither and Pareigis [GP87] showed how all Hopf-Galois structures on a finite separable field extension $L / K$ can be described in terms of group theory. We consider here only the case when $L / K$ is a Galois extension in the classical sense (i.e., normal as well as separable). Let $\Gamma=\operatorname{Gal}(L / K)$. Then the Hopf-Galois structures on $L / K$ correspond bijectively to the regular subgroups $G \subset \operatorname{Perm}(\Gamma)$ which are normalised by the group $\lambda(\Gamma)$ of left translations by $\Gamma$. Here Perm $(\Gamma)$ is the group of permutations of the underlying set of $\Gamma$. The Hopf algebra acting on $L$ in the Hopf-Galois structure corresponding to $G$ is $H=L[G]^{\Gamma}$, the algebra of $\Gamma$-fixed points of $L[G]$, where $\Gamma$ acts simultaneously on $L$ as field automorphisms and on $G$ as conjugation by left translations inside $\operatorname{Perm}(\Gamma)$. The type of a Hopf-Galois structure is by definition the isomorphism type of the corresponding group $G$.

Given abstract groups $\Gamma$ and $G$ of the same (finite) order, we denote by $e(\Gamma, G)$ the number of Hopf-Galois structures of type $G$ on a Galois extension $L / K$ with $\operatorname{Gal}(L / K) \cong \Gamma$. By the theorem of Greither and Pareigis, this is just the number of regular subgroups of $\operatorname{Perm}(\Gamma)$ which are isomorphic to $G$ and are normalised by $\lambda(\Gamma)$. Equivalently, $e(\Gamma, G)$ is the number of $\operatorname{Aut}(G)$-orbits of regular embeddings $\alpha: G \rightarrow \operatorname{Perm}(\Gamma)$ whose image $\alpha(G)$ is normalised by $\lambda(\Gamma)$. As shown in [Byo96], there is a bijection between the regular embeddings $\alpha: G \rightarrow \operatorname{Perm}(\Gamma)$ and the regular embeddings $\beta: \Gamma \rightarrow \operatorname{Perm}(G)$, under which $\alpha(G)$ is normalised by $\lambda(\Gamma)$ if and only of $\beta(\Gamma)$ is contained in the normaliser $\operatorname{Hol}(G)=$ $\lambda(G) \rtimes \operatorname{Aut}(G)$ of $G$ in $\operatorname{Perm}(G)$. Thus $e(\Gamma, G)$ is the number of $\operatorname{Aut}(G)$ orbits of regular embeddings $\Gamma \rightarrow \operatorname{Hol}(G)$, or, equivalently,

$$
e(\Gamma, G)=\frac{|\operatorname{Aut}(\Gamma)|}{|\operatorname{Aut}(G)|} e^{\prime}(\Gamma, G)
$$

where $e^{\prime}(\Gamma, G)$ is the number of regular subgroups of $\operatorname{Hol}(G)$ isomorphic to $\Gamma$. As $\operatorname{Hol}(G)$ is usually much smaller than $\operatorname{Perm}(\Gamma)$, it is in practice easier to calculate $e(\Gamma, G)$ by working in $\operatorname{Hol}(G)$ and using (3.1), rather than by working directly in $\operatorname{Perm}(\Gamma)$.

\section{The holomorph of $G$}

Let $G=G(d, e, k)$ be a group of squarefree order $n=d e$ as in Lemma 2.1. In this section, we recall from [AB18] some results on $G, \operatorname{Aut}(G)$ and $\operatorname{Hol}(G)$.

By [AB18, Prop. 3.5], the centre $Z(G)$ and commutator subgroup $G^{\prime}$ of $G$ are respectively the cyclic groups $\left\langle\sigma^{g}\right\rangle$ of order $z$ and $\left\langle\sigma^{z}\right\rangle$ of order $g$, where $z, g$ are defined in (2.1). 
We write elements of $\operatorname{Hol}(G)=G \rtimes \operatorname{Aut}(G)$ as $[x, \alpha]$, where $x \in G$ and $\alpha \in \operatorname{Aut}(G)$. The multiplication in $\operatorname{Hol}(G)$ is given by

$$
[x, \alpha]\left[x^{\prime}, \alpha^{\prime}\right]=\left[x \alpha\left(x^{\prime}\right), \alpha \alpha^{\prime}\right],
$$

and the action of $\operatorname{Hol}(G)$ on $G$ is given by

$$
[x, \alpha] \cdot y=x \alpha(y) \text {. }
$$

Thus the normal subgroup $G$ in $\operatorname{Hol}(G)=G \rtimes \operatorname{Aut}(G)$ identifies as the group of left translations in $\operatorname{Perm}(G)$.

In [AB18, Lemma 4.1] we determined $\operatorname{Aut}(G)$ :

Lemma 4.1. $\operatorname{Aut}(G) \cong \mathbb{Z}_{g} \rtimes \mathbb{Z}_{e}^{\times}$, and $\operatorname{Aut}(G)$ is generated by $\theta$ and by $\phi_{s}$ for $s \in \mathbb{Z}_{e}^{\times}$where

$$
\theta(\sigma)=\sigma, \quad \theta(\tau)=\sigma^{z} \tau
$$

and

$$
\phi_{s}(\sigma)=\sigma^{s}, \quad \phi_{s}(\tau)=\tau .
$$

These satisfy the relations

$$
\theta^{g}=\operatorname{id}_{G}, \quad \phi_{s} \phi_{t}=\phi_{s t}, \quad \phi_{s} \theta \phi_{s}^{-1}=\theta^{s} .
$$

An arbitrary element of $\operatorname{Hol}(G)$ therefore has the form $\left[\sigma^{u} \tau^{f}, \theta^{v} \phi_{t}\right]$ for $u \in \mathbb{Z}_{e}, f \in \mathbb{Z}_{d}, v \in \mathbb{Z}_{g}$ and $t \in \mathbb{Z}_{e}^{\times}$. We record some consequences of this.

\section{Proposition 4.2 .}

(i) The map $\operatorname{Hol}(G) \rightarrow\left\{\phi_{s}: s \in \mathbb{Z}_{e}^{\times}\right\} \cong \mathbb{Z}_{e}^{\times}$, given by $\left[\sigma^{u} \tau^{f}, \theta_{v} \phi_{t}\right] \mapsto$ $\phi_{t}$, is a group homomorphism.

(ii) The map $\operatorname{Hol}(G) \rightarrow\langle\tau\rangle \cong \mathbb{Z}_{d}$, given by $\left[\sigma^{u} \tau^{f}, \theta_{v} \phi_{t}\right] \mapsto \tau^{f}$, is a group homomorphism

Proof. (i) The map is the composite of the two quotient homomorphisms $\operatorname{Hol}(G) \rightarrow \operatorname{Aut}(G) \rightarrow \mathbb{Z}_{e}^{\times}$.

(ii) This follows from the fact that every automorphism of $G$ induces the trivial automorphism on the quotient $G /\langle\sigma\rangle \cong \mathbb{Z}_{d}$ of $G$, as explained in the proof of $\left[\mathrm{AB} 18\right.$, Prop. 4.3]. (Note that the map $\left[\sigma^{u} \tau^{f}, \theta^{v} \phi_{t}\right] \mapsto \sigma^{u} \tau^{f}$ is not a homomorphism.)

In the next section, we will consider pairs of elements of $\operatorname{Hol}(G)$ of the following special form:

$$
X=\left[\sigma^{a}, \theta^{c}\right], \quad Y=\left[\sigma^{u} \tau, \theta^{v} \phi_{t}\right]
$$

Thus $X$ does not involve $\tau$ or any $\phi_{s}$, and $\tau$ occurs in $Y$ with exponent 1 .

As $\theta(\sigma)=\sigma$, it is clear that

$$
X^{j}=\left[\sigma^{a j}, \theta^{c j}\right], \quad X^{j} \cdot \sigma^{i}=\sigma^{a j+i} \text { for } i, j \geq 0 .
$$


Lemma 4.3. For $Y$ as in (4.2) and $j \geq 0$, we have

$$
Y^{j}=\left[\sigma^{A(j)} \tau^{j}, \theta^{v S(t, j)} \phi_{t^{j}}\right]
$$

where

$$
A(j)=u S(t k, j)+v z k T(k, t, j),
$$

with

$$
S(m, j)=\sum_{i=0}^{j-1} m^{i}
$$

and

$$
T(k, t, j)=\sum_{h=0}^{j-1} S(t, h) k^{h-1} \text { for } j \geq 1, \quad T(k, t, 0)=0 .
$$

Proof. See [AB18, Lemma 4.2].

Using the fact that $k^{d} \equiv 1(\bmod e)$, we can determine the residue classes of the sums in Lemma 4.3 at primes dividing $e$ in the case that $j$ is a multiple of $d$.

Proposition 4.4. Let $q$ be a prime dividing e. Then we have the following congruences mod $q$. (We omit the modulus for brevity.)

(i) For any $s, i \in \mathbb{Z}$ with $i \geq 0$, we have

$$
S(s, d i) \equiv \begin{cases}d i & \text { if } s \equiv 1 \\ \frac{s^{d i}-1}{s-1} & \text { otherwise. }\end{cases}
$$

(ii) If $k \not \equiv 1$ then, for any $t, i \in \mathbb{Z}$ with $i \geq 0$, we have

$$
T(k, t, d i) \equiv \begin{cases}\frac{d i}{k(k-1)} & \text { if } t \equiv 1 ; \\ \frac{d i}{k(t-1)} & \text { if } t k \equiv 1 ; \\ \frac{\left(t^{d i}-1\right)}{k(t-1)(t k-1)} & \text { otherwise. }\end{cases}
$$

Proof. This is [AB18, Prop. 5.2]. 


\section{Regular subgroups in $\operatorname{Hol}(G)$}

As before, let $G=G(d, e, k)$ be a group of squarefree order $n$ as in Lemma 2.1, and let $\Gamma=G(\delta, \epsilon, \kappa)$ be a second group of order $n$, as in (2.2). In this section, we begin our investigation of regular subgroups of $\operatorname{Hol}(G)$ isomorphic to $\Gamma$.

It will be convenient to modify the presentation (2.2) of $\Gamma$. Set $X=s^{\zeta}$ and $Y=t s^{\gamma}$. As $s^{\gamma}$ generates the centre of $\Gamma$, we have

$$
\Gamma=\left\langle X, Y: X^{\gamma}=Y^{\zeta \delta}=1, Y X Y^{-1}=X^{\kappa}\right\rangle \text {. }
$$

Remark 5.1. In (5.1), $\kappa$ is viewed as an element of $\mathbb{Z}_{\gamma}^{\times}$such that $\operatorname{gcd}(\kappa-1, \gamma)=1$. This is equivalent to viewing $\kappa$ as an element of $\mathbb{Z}_{\epsilon}^{\times} \cong \mathbb{Z}_{\gamma}^{\times} \times \mathbb{Z}_{\zeta}^{\times}$with $\operatorname{gcd}(\kappa-1, \epsilon)=\zeta$, as in $(2.2)$ and $(2.3)$.

Proposition 5.2. If $\operatorname{Hol}(G)$ contains a regular subgroup $\Gamma^{*} \cong \Gamma$ then $\gamma \mid$ e. Moreover, if $X$ and $Y$ are generators of $\Gamma^{*}$ satisfying the relations in (5.1) then the subgroup $\left\langle X, Y^{d}\right\rangle$ of $\Gamma^{*}$ of order $e$ acts regularly on the subset $\left\{\sigma^{m}: m \in \mathbb{Z}\right\}$ of $G$.

Proof. Let $\Gamma^{*}=\langle X, Y\rangle \cong \Gamma$ be a regular subgroup, where the generators $X, Y$ satisfy the relations in (5.1). Then the commutator subgroup of $\Gamma^{*}$ is generated by $X$. Thus $X$ lies in the commutator subgroup of $\operatorname{Hol}(G)$, and therefore in the kernel of the homomorphism $\operatorname{Hol}(G) \rightarrow\langle\tau\rangle$ of Proposition 4.2(ii). Hence $X$ cannot involve $\tau$. But $\Gamma^{*}$ is regular, so $X^{i} Y^{f} \cdot 1_{G}=\tau$ for some $i, f$. It follows that $Y^{f}=\left[\sigma^{a} \tau, \psi\right]$ for some $a \in \mathbb{Z}$ and some $\psi \in \operatorname{Aut}(G)$. Thus, for $j \geq 0$, we have $Y^{f j} \cdot 1_{G} \in\left\{\sigma^{m}: m \in \mathbb{Z}\right\}$ if and only if $d \mid j$. As $Y$ has order $\zeta \delta$, it follows that $d \mid \zeta \delta$. Since $d e=n=\delta \gamma \zeta$, this is equivalent to $\gamma \mid e$. Moreover, the subgroup $\left\langle X, Y^{d}\right\rangle$ has order $e$ and acts without fixed points on the subset $\left\{\sigma^{m}: m \in \mathbb{Z}\right\}$ of $G$, which has cardinality $e$. Hence this action is regular.

In view of the first assertion of Proposition 5.2, we now impose the following hypothesis:

Hypothesis 5.3. The groups $G$ and $\Gamma$ are chosen so that $\gamma \mid e$.

Note that, as shown above, the condition $\gamma \mid e$ is equivalent to $d \mid \zeta \delta$.

Recall that in $\S 2$ we defined the set $\mathcal{K}$ and the group $\Delta$, and chose a system $\kappa_{1}, \ldots, \kappa_{w}$ of orbit representatives for the action of $\Delta$ on $\mathcal{K}$, with $w=\varphi(\operatorname{gcd}(\delta, d))$.

Remark 5.4. If $\operatorname{gcd}(\delta, d)=1$ or 2 , then $w=1$ and we may take $\kappa_{1}=\kappa$. If $\operatorname{gcd}(\delta, d)>2$ then $w$ is even and $-1 \notin \Delta$. We may then choose the $\kappa_{h}$ so that $\kappa_{w+1-h}=\kappa_{h}^{-1}$ for $1 \leq h \leq w$. 
Lemma 5.5. Let $\Gamma^{*}$ be a regular subgroup of $\operatorname{Hol}(G)$ isomorphic to $\Gamma$. Then there is a unique $h$ with $1 \leq h \leq w$ such that $\Gamma^{*}$ is generated by a pair of elements $X, Y$ in the special form of (4.2) which satisfy the relations

$$
\begin{gathered}
X^{\gamma}=Y^{\zeta \delta}=1, \\
Y X Y^{-1}=X^{\kappa_{h}} .
\end{gathered}
$$

Indeed, $\Gamma^{*}$ contains exactly $\gamma \varphi(e) w / \varphi(\delta)$ such pairs of generators.

Proof. Since $\Gamma^{*} \cong \Gamma$, we can find some pair of generators $X, Y$ satisfying the relations in (5.1). As in the proof of Proposition 5.2, $\tau$ cannot occur in $X$, and there is some $f \in \mathbb{Z}$ so that $\tau$ occurs with exponent 1 in $Y^{f}$. Then $\operatorname{gcd}(f, d)=1$. Since $d \mid \zeta \delta$ and $\zeta \delta$ is squarefree, we may choose $f$ with $\operatorname{gcd}(f, \zeta \delta)=1$. Thus $Y^{f}$ has order $\zeta \delta$. We replace $Y$ by $Y^{f}$. Our new pair of generators $X, Y$ satisfy the relations in (5.1), but with $\kappa$ replaced by $\kappa^{f}$. Moreover, $Y$ is as in (4.2) and $X$ has the form

$$
X=\left[\sigma^{a}, \theta^{c} \phi_{s}\right] \text {. }
$$

We claim that $s \equiv 1(\bmod e)$, so that $X$ is also as in (4.2). Applying Proposition 4.2(i) to the relations $X^{\gamma}=1, Y X=X^{\kappa} Y$, we obtain $\phi_{s}^{\gamma}=1, \phi_{t} \phi_{s}=\phi_{s}^{\kappa} \phi_{t}$. Thus $s^{\gamma} \equiv 1 \equiv s^{\kappa-1}(\bmod e) \cdot \operatorname{As} \operatorname{gcd}(\gamma, \kappa-1)=$ 1 , it follows that $s \equiv 1(\bmod e)$ as claimed.

We next consider further changes to our generators $X, Y$ so that they are still of the form (4.2), and satisfy the relations in (5.1) except that $\kappa$ may be replaced by some other element of $\mathcal{K}$. Thus we can replace $X$ by $x=X^{i}$ for any $i \in \mathbb{Z}_{\gamma}^{\times}$, and $Y$ by $y=X^{j} Y^{m}$ for any $j \in \mathbb{Z}_{\gamma}$ and $m \in \mathbb{Z}_{\zeta \delta}^{\times}$with $m \equiv 1(\bmod d)$. (The last condition ensures that $\tau$ still occurs with exponent 1 in $y$.) Then

$$
y x y^{-1}=\left(X^{j} Y^{m}\right) X^{i}\left(Y^{-m} X^{-j}\right)=X^{i \kappa^{m}}=x^{\kappa^{m}}
$$

As $\kappa \in \mathbb{Z}_{\gamma}^{\times}$has order $\delta$, we have

$$
\begin{aligned}
\left\{\kappa^{m}: m \equiv 1 \quad(\bmod d)\right\} & =\left\{\kappa^{r}: r \equiv 1 \quad(\bmod \operatorname{gcd}(\delta, d))\right\} \\
& =\left\{\kappa^{r}: r \in \Delta\right\} .
\end{aligned}
$$

Thus, given $r \in \mathbb{Z}_{\delta}^{\times}$, the original generators $X, Y$ can be replaced by generators $x, y$ of the form (4.2) satisfying the relations $x^{\gamma}=y^{\zeta \delta}=1$, $y x=x^{\kappa^{r}} y$ if and only if $r \in \Delta$. It follows that there is a unique $h$ such that $\Gamma^{*}$ contains a pair of generators satisfying (4.2), (5.2) and (5.3).

Finally, suppose $X, Y$ is one such pair of generators. We determine when $x=X^{i}, y=X^{j} Y^{m}$ is another. Since $X$ has order $\gamma$, (5.3) will hold if and only if $\kappa^{m} \equiv \kappa(\bmod \gamma)$. As $\kappa$ has order $\delta$, this is equivalent to $m \equiv 1(\bmod \delta)$. Since we already have the condition 
$m \equiv 1(\bmod d)$, and $d \mid \zeta \delta$ by Hypothesis 5.3 , we must choose $m \in \mathbb{Z}_{\zeta \delta}^{\times}$ with $m \equiv 1(\bmod \operatorname{lcm}(\delta, d))$. Hence the number of choices for $m$ is $\varphi(\zeta \delta) / \varphi(\operatorname{lcm}(\delta, d))$. Now, since $d$ and $\delta$ are squarefree, we have

$$
\varphi(\operatorname{lcm}(\delta, d))=\varphi\left(\frac{d \delta}{\operatorname{gcd}(\delta, d)}\right)=\frac{\varphi(d) \varphi(\delta)}{\varphi(\operatorname{gcd}(\delta, d))}=\frac{\varphi(d) \varphi(\delta)}{w} .
$$

So the number of choices for $m$ is $\varphi(\zeta \delta) w /(\varphi(d) \varphi(\delta))$. There are $\varphi(\gamma)$ choices for $i \in \mathbb{Z}_{\gamma}^{\times}$and $\gamma$ choices for $j \in \mathbb{Z}_{\gamma}$. Therefore, the number of pairs $X, Y$ of generators of $\Gamma^{*}$ satisfying (4.2), (5.2) and (5.3) is

$$
\frac{\varphi(\gamma) \gamma \varphi(\zeta \delta) w}{\varphi(d) \varphi(\delta)}=\frac{\gamma \varphi(n) w}{\varphi(d) \varphi(\delta)}=\frac{\gamma \varphi(e) w}{\varphi(\delta)}
$$

Lemma 5.5 shows that the regular subgroups of $\operatorname{Hol}(G)$ isomorphic to $\Gamma$ fall into $w$ disjoint families $\mathcal{F}_{1}, \ldots, \mathcal{F}_{w}$, where $\mathcal{F}_{h}$ consists of those subgroups with a pair of generators $X, Y$ satisfying (4.2) and (5.2), and satisfying (5.3) for the orbit representative $\kappa_{h}$. These families therefore correspond to the orbits of $\Delta$ on $\mathcal{K}$. (We will see in Lemma 7.1 that all the $\mathcal{F}_{h}$ are nonempty.) However, not every pair of elements $(X, Y)$ satisfying (4.2), (5.2) and (5.3) will generate a regular subgroup. We now characterise those that do.

Lemma 5.6. Let $1 \leq h \leq w$ and let $X, Y \in \operatorname{Hol}(G)$ be as in (4.2). Suppose further that $X$ and $Y$ satisfy (5.3). Then the group $\langle X, Y\rangle \subseteq$ $\mathrm{Hol}(G)$ is regular on $G$ if and only if

(i) the group $\langle X\rangle$ acts regularly on the subset $\left\{\sigma^{e m / \gamma}: m \in \mathbb{Z}\right\}$ of $G$ of cardinality $\gamma$

(ii) the group $\left\langle X, Y^{d}\right\rangle$ acts transitively on the subset $\left\{\sigma^{m}: m \in \mathbb{Z}\right\}$ of $G$ of cardinality e;

(iii) $Y^{\zeta \delta}=1$.

If these conditions hold, then (5.2) also holds, and $\langle X, Y\rangle \cong \Gamma$.

Proof. Suppose $\Gamma^{*}=\langle X, Y\rangle$ is regular. Then (i) follows from Proposition 5.2, so in particular $X$ has order $\gamma$. As $\left|\Gamma^{*}\right|=n=\gamma \zeta \delta$, (iii) follows. Since $Y^{d}$ does not involve $\tau$, the subgroup $\left\langle X, Y^{d}\right\rangle$ of index $d$ acts on $\left\{\sigma^{m}: m \in \mathbb{Z}\right\}$. This action must be regular, and hence transitive. Thus (ii) holds.

Conversely, suppose $X, Y$ satisfy (i), (ii) and (iii), and let $\Gamma^{*}=$ $\langle X, Y\rangle$. By (i), $X$ has order $\gamma$. It then follows from (iii) that (5.2) is satisfied. In particular, $\Gamma^{*}$ has order dividing $n$. We claim that $\Gamma^{*}$ is transitive on $G$. This will show that $\Gamma^{*}$ is regular and isomorphic to $\Gamma$. Let $\sigma^{i} \tau^{j}$ be an arbitrary element of $G$. As $\tau$ occurs in $Y$ with exponent 
1, we have $Y^{-j} \cdot \sigma^{i} \tau^{j}=\sigma^{m}$ for some $m$. It follows from (ii) that $\sigma^{i} \tau^{j}$ is in the same $\Gamma^{*}$-orbit as $1_{G}$. Hence $\Gamma^{*}$ is transitive as claimed.

Definition 5.7. For $1 \leq h \leq w$, let

$$
\mathcal{N}_{h} \subset \mathbb{Z}_{e}^{\times} \times \mathbb{Z}_{e} \times \mathbb{Z}_{g} \times \mathbb{Z}_{e} \times \mathbb{Z}_{g}
$$

be the set of quintuples $(t, a, c, u, v)$ such that the corresponding elements $X=\left[\sigma^{a}, \theta^{c}\right], Y=\left[\sigma^{u} \tau, \theta^{v} \phi_{t}\right]$ satisfy (5.3) and generate a regular subgroup isomorphic to $\Gamma$.

Thus $(t, a, c, u, v) \in \mathcal{N}_{h}$ if and only if $X$ and $Y$ satisfy (5.3) and conditions (i), (ii), (iii) of Lemma 5.6.

By the last assertion of Lemma 5.5, the number $e^{\prime}(\Gamma, G)$ of regular subgroups isomorphic to $\Gamma$ in $\operatorname{Hol}(G)$ is given by

$$
e^{\prime}(\Gamma, G)=\sum_{h=1}^{w}\left|\mathcal{F}_{h}\right|=\frac{\varphi(\delta)}{\gamma \varphi(e) w} \sum_{h=1}^{w}\left|\mathcal{N}_{h}\right| \text {. }
$$

\section{Quintuples GIVING REgulaR SUbGROUPS}

Suppose that Hypothesis 5.3 holds. Our goal in this section is to give an explicit characterisation of the set $\mathcal{N}_{h}$ in Definition 5.7 by means of congruences at each prime $q \mid e$. We will achieve this in Lemma 6.12, after defining some further notation and proving a number of preliminary propositions.

Recall that $r_{q}=\operatorname{ord}_{q}(k)$ for each prime $q \mid e$, and $\rho_{q}=\operatorname{ord}_{q}(\kappa)$ for each $q \mid \epsilon$. Thus we have

$$
r_{q}=1 \Leftrightarrow q\left|z, \quad r_{q}\right| \operatorname{gcd}(d, q-1), \quad \operatorname{lcm}\left\{r_{q}: q \mid g\right\}=d,
$$

and similarly for the $\rho_{q}$.

Remark 6.1. If $q \mid g$, we have $1<r_{q} \leq q-1$, so $q \geq 3$. Thus $g$ is always odd.

We divide the set of primes $q \mid e$ into six subsets (any of which may be empty).

\section{Definition 6.2.}

$$
\begin{gathered}
P=\{\text { primes } q \mid \operatorname{gcd}(\gamma, z)\} \\
Q=\{\text { primes } q \mid \operatorname{gcd}(\zeta \delta, z)\} \\
R=\left\{\text { primes } q \mid \operatorname{gcd}(\gamma, g): \rho_{q} \neq r_{q}\right\} \\
S=\left\{\text { primes } q \mid \operatorname{gcd}(\gamma, g): \rho_{q}=r_{q}>2\right\} \\
T=\left\{\text { primes } q \mid \operatorname{gcd}(\gamma, g): \rho_{q}=r_{q}=2\right\} \\
U=\{\text { primes } q \mid \operatorname{gcd}(\zeta \delta, g)\}
\end{gathered}
$$


For $1 \leq h \leq w$, we further define some subsets of $S$ depending on $h$.

\section{Definition 6.3.}

$$
\begin{gathered}
S_{h}^{+}=\left\{q \in S: \kappa_{h} \equiv k \quad(\bmod q)\right\} \\
S_{h}^{-}=\left\{q \in S: \kappa_{h} \equiv k^{-1} \quad(\bmod q)\right\} \\
S_{h}=S_{h}^{+} \cup S_{h}^{-} \\
S_{h}^{\prime}=S \backslash S_{h}
\end{gathered}
$$

The sets $S, T$ and $S_{h}$ are as already defined in $\S 2$.

Remark 6.4. Let $q \in S$. Then, as $\rho_{q} \mid \operatorname{gcd}(\delta, d)$, we have $\kappa_{h}^{m} \equiv \kappa_{h}$ $(\bmod q)$ for $m \equiv 1(\bmod \operatorname{gcd}(\delta, d))$. This shows that the sets $S_{h}^{+}$and $S_{h}^{-}$are independent of the choice of orbit representatives $\kappa_{h}$. Also, $q \in T$ if and only if $k \equiv \kappa \equiv-1 \not \equiv 1(\bmod q)$. In particular, $T=\emptyset$ unless $d$ and $\delta$ are both even.

Remark 6.5. If $w>1$ and the $\kappa_{h}$ are chosen as in Remark 5.4, then $S_{h}^{-}=S_{w+1-h}^{+}$for each $h$.

The set $S$ is the union of the sets $S_{h}^{+}$, but in general this union is not disjoint.

Proposition 6.6. Let $q \in S$. Then there are exactly $w / \varphi\left(r_{q}\right)$ values of $h$ with $q \in S_{h}^{+}$. In particular, $q \in S_{h}^{+}$for a unique $h$ if and only if $r_{q}=\operatorname{gcd}(\delta, d)$ or $r_{q}=\frac{1}{2} \operatorname{gcd}(\delta, d)$.

Proof. We may decompose the group $\mathbb{Z}_{\delta}^{\times}$as

$$
\mathbb{Z}_{\delta}^{\times}=\mathbb{Z}_{\operatorname{gcd}(\delta, d)}^{\times} \times \mathbb{Z}_{\operatorname{gcd}(\delta, e)}^{\times} \cong \mathbb{Z}_{\operatorname{gcd}(\delta, d)}^{\times} \times \Delta,
$$

so that the orbits of $\mathcal{K}$ under $\Delta$ correspond to the elements of $\mathbb{Z}_{\operatorname{gcd}(\delta, d)}^{\times}$. Explicitly, if the orbit represented by $\kappa_{h}$ corresponds to $j \in \mathbb{Z}_{\operatorname{gcd}(\delta, d)}^{\times}$, then $\kappa_{h} \equiv \kappa^{J}(\bmod \epsilon)$ for some $J \equiv j(\bmod \operatorname{gcd}(\delta, d))$ with $\operatorname{gcd}(J, \delta)=$ 1. Now let also $\kappa_{h^{\prime}} \equiv \kappa^{J^{\prime}}$ represent the orbit corresponding to $j^{\prime}$. Then

$$
\begin{aligned}
\kappa_{h} \equiv \kappa_{h^{\prime}} \quad(\bmod q) & \Leftrightarrow \kappa^{J} \equiv \kappa^{J^{\prime}} \quad(\bmod q) \\
& \Leftrightarrow J \equiv J^{\prime} \quad\left(\bmod \rho_{q}\right) \\
& \Leftrightarrow j \equiv j^{\prime} \quad\left(\bmod \rho_{q}\right)
\end{aligned}
$$

since $r_{q}=\rho_{q}$ divides $\operatorname{gcd}(\delta, d)$. As the canonical surjection $\mathbb{Z}_{\operatorname{gcd}(\delta, d)}^{\times} \rightarrow$ $\mathbb{Z}_{r_{q}}^{\times}$has kernel of cardinality $w / \varphi\left(r_{q}\right)$, it follows that $j \equiv j^{\prime}\left(\bmod r_{q}\right)$ for precisely $w / \varphi\left(r_{q}\right)$ of the $w$ possible values of $j^{\prime}$. Thus $q \in S_{h}^{+}$for precisely $w / \varphi\left(r_{q}\right)$ values of $h$.

In particular, as $w=\varphi(\operatorname{gcd}(\delta, d))$, there is a unique $h$ with $q \in S_{h}^{+}$ if and only if $\varphi\left(r_{q}\right)=\varphi(\operatorname{gcd}(\delta, d))$. As $r_{q} \mid \operatorname{gcd}(\delta, d)$, and $\operatorname{gcd}(\delta, d)$ is squarefree, this occurs if and only if $\operatorname{gcd}(\delta, d)=r_{q}$ or $2 r_{q}$. 
We now fix $h \in\{1, \ldots, w\}$. To simplify the notation, we write $\kappa$ in place of $\kappa_{h}$ for the rest of this section.

Let

$$
(t, a, c, u, v) \in \mathbb{Z}_{e}^{\times} \times \mathbb{Z}_{e} \times \mathbb{Z}_{g} \times \mathbb{Z}_{e} \times \mathbb{Z}_{g} .
$$

We wish to determine when this quintuple belongs to $\mathcal{N}_{h}$. Equivalently, we wish to determine when the elements $X=\left[\sigma^{a}, \theta^{c}\right]$ and $Y=\left[\sigma^{u} \tau, \theta^{v} \phi_{t}\right]$ of $\operatorname{Hol}(G)$ generate a subgroup $\Gamma^{*}=\langle X, Y\rangle$ in $\mathcal{F}_{h}$.

We therefore need to find the restrictions on $(t, a, c, u, v)$ which ensure that (5.3) holds for the value of $\kappa=\kappa_{h}$ corresponding to our choice of $h$, and also that conditions (i)-(iii) of Lemma 5.6 hold. To do this, we translate these four conditions into congruences on $t, a, c, u, v$ modulo each prime $q$ dividing $e$. We successively impose the conditions Lemma 5.6(i), (5.3), Lemma 5.6(ii) and Lemma 5.6(iii), in that order, recording the resulting congruences in Propositions 6.7, 6.8, 6.9 and 6.11 respectively. We then summarise these congruences in Lemma 6.12 .

In the following, all congruences are modulo the relevant prime $q$ unless otherwise indicated.

Proposition 6.7. Condition (i) of Lemma 5.6 is equivalent to the following conditions at each prime $q \mid e$ :

$$
\begin{gathered}
\text { if } q \mid \gamma \text { then } a \neq 0 ; \\
\text { if } q \mid \operatorname{gcd}(\zeta \delta, e) \text { then } a \equiv 0 ; \\
\text { if } q \mid \operatorname{gcd}(\zeta \delta, g) \text { then } c \equiv 0 \text {. }
\end{gathered}
$$

Proof. Condition (i) of Lemma 5.6 is equivalent to

$$
X^{j} \cdot 1_{G}=1_{G} \Leftrightarrow \gamma \mid j, \quad X^{\gamma}=1
$$

and so, using (4.3), to

$$
e|a j \Leftrightarrow \gamma| j, \quad c \gamma \equiv 0 \quad(\bmod g) .
$$

(Recall that $\gamma \mid e$ by Hypothesis 5.3). These in turn are equivalent to (6.1), (6.2) and (6.3).

Proposition 6.8. Assume that condition (i) of Lemma 5.6 holds. Then (5.3) is equivalent to the following conditions for each prime q:

$$
\text { if } q \mid \operatorname{gcd}(\gamma, z) \text { then } t \equiv \kappa \text {; }
$$

if $q \mid \operatorname{gcd}(\gamma, g)$ then either

(i) $t \equiv \kappa, \quad c \equiv \lambda a$, or

(ii) $t k \equiv \kappa, \quad c \equiv 0$, 
where $\lambda=z^{-1}(k-1) \in \mathbb{Z}_{g}^{\times}$.

Proof. Our assumption means that (6.1), (6.2), (6.3) hold.

Writing (5.3) as $Y X=X^{\kappa} Y$ and expanding using (4.1) and (4.3), we obtain

$$
\left[\sigma^{u+a t k} \tau, \theta^{v+c t} \phi_{t}\right]=\left[\sigma^{a \kappa+u+z c \kappa} \tau, \theta^{c \kappa+v} \phi_{t}\right] .
$$

This is equivalent to the two congruences

$$
\begin{gathered}
a(t k-\kappa) \equiv z c \kappa \quad(\bmod e), \\
c(t-\kappa) \equiv 0 \quad(\bmod g) .
\end{gathered}
$$

Note that, although $\kappa$ is only defined $\bmod \epsilon,(6.6)$ and (6.7) make sense because of (6.2) and (6.3). We determine when (6.6) and (6.7) hold $\bmod q$ for each prime $q \mid e$.

If $q \mid \zeta \delta$ then (6.6) and (6.7) are automatically satisfied because of (6.2), (6.3).

If $q \mid \operatorname{gcd}(\gamma, z)$ then (6.7) gives no condition $\bmod q$ and (6.6) reduces to $a(\kappa-t) \equiv 0$ since $k \equiv 1$. By (6.1), this is the same as (6.4).

Finally, suppose that $q \mid \operatorname{gcd}(\gamma, g)$. If $t \equiv \kappa$ then (6.7) holds for any choice of $c$, and, substituting into (6.6), we get (6.5)(i). If $t \not \equiv \kappa$ then (6.7) gives $c \equiv 0$, and then (6.6) reduces to $t k \equiv \kappa$, again using (6.1). This gives $(6.5)$ (ii).

Recall from Lemma 4.3 that $Y^{j}=\left[\sigma^{A(j)} \tau^{j}, \theta^{v S(t, j)} \phi_{t^{j}}\right]$ where

$$
A(j)=u S(t k, j)+v z k T(k, t, j),
$$

with

$$
S(m, j)=\sum_{i=0}^{j-1} m^{i}
$$

and

$$
T(k, t, j)=\sum_{h=0}^{j-1} S(t, h) k^{h-1} \text { for } j \geq 1, \quad T(k, t, 0)=0 .
$$

Proposition 6.9. Suppose that (5.3) and condition (i) of Lemma 5.6 hold. Then condition (ii) of Lemma 5.6 is equivalent to

(6.8) $A(d i)$ represents all residue classes $\bmod \operatorname{gcd}(\zeta \delta, e)$ as $i$ varies.

Moreover, this occurs if and only if the following two conditions hold:

$$
\text { for } q \mid \operatorname{gcd}(\zeta \delta, z) \text { we have } t \equiv 1 \text { and } u \not \equiv 0
$$




$$
\text { for } q \mid \operatorname{gcd}(\zeta \delta, g) \text { we have either }
$$

$$
\begin{aligned}
& \text { (i) } t \equiv 1, \quad v \neq \equiv 0, \text { or } \\
& \text { (ii) } t k \equiv 1, \quad v \neq \equiv \mu u,
\end{aligned}
$$

where $\mu=k^{-1} z^{-1}(k-1) \in \mathbb{Z}_{g}^{\times}$.

Proof. We have $Y^{d i} \cdot 1_{G}=\sigma^{A(d i)}$ for $i \geq 0$. On the other hand, it follows from (4.3), (6.1) and (6.2) that, for any $j \in \mathbb{Z}$, the orbit of $\sigma^{j}$ under $\langle X\rangle$ is $\left\{\sigma^{j+e m / \gamma}: m \in \mathbb{Z}\right\}$. Thus condition (ii) of Lemma 5.6 holds if and only if $A(d i)$ represents all residue classes $\bmod e / \gamma=\operatorname{gcd}(\zeta \delta, e)$ as $i$ varies. This proves the first assertion.

Suppose that (6.8) holds. Then $A(d i)$ represents all residue classes $\bmod q$ for each prime $q \mid \operatorname{gcd}(\zeta \delta, e)$. We analyse this condition, distinguishing several cases. We have

$$
A(d i)=u S(t k, d i)+v z k T(k, t, d i)
$$

First let $q \mid \operatorname{gcd}(\zeta \delta, z)$. Then $k \equiv 1$, and we have $A(d i) \equiv u S(t, d i)$. If $t \not \equiv 1$ then, by Proposition 4.4(i),

$$
A(d i) \equiv \frac{u\left(t^{d i}-1\right)}{t-1} .
$$

As there is no $i$ with $t^{d i} \equiv 0$, this cannot take all values mod $q$. If $t \equiv 1$, we have $A(d i) \equiv u d i$, which takes all values $\bmod q$ if and only if $u \not \equiv 0$. This gives $(6.9)$.

Now let $q \mid \operatorname{gcd}(\zeta \delta, g)$. Thus $k \not \equiv 1$, but of course $k^{d} \equiv 1$. If $t \not \equiv 1 \not \equiv t k$ then, as $(t k)^{d} \equiv t^{d}$, it follows from Proposition 4.4 that each of the two terms of $A(d i)$ is the product of $t^{d i}-1$ by a term independent of $i$. Thus, as above, $A(d i)$ cannot represent all residue classes $\bmod q$.

If $q \mid \operatorname{gcd}(\zeta \delta, g)$ and $t \equiv 1$, we have

$$
S(t k, d i) \equiv S(k, d i) \equiv \frac{k^{d i}-1}{k-1} \equiv 0,
$$

which by Proposition 4.4(ii) gives

$$
A(d i) \equiv v z k T(k, 1, d i) \equiv \frac{v z d i}{k-1} .
$$

This represents all residue classes $\bmod q$ if and only if $v \not \equiv 0$, giving (6.10)(i).

If $q \mid \operatorname{gcd}(\zeta \delta, g)$ and $t k \equiv 1$ then

$$
A(d i) \equiv u d i+\frac{v z k d i}{k(t-1)} \equiv \frac{z k}{k-1}(\mu u-v) d i,
$$


which represents all residue classes $\bmod q$ if and only if $v \not \equiv \mu u$. This gives (6.10)(ii).

We have now shown that if (6.8) holds then (6.9) or (6.10) holds for each prime $q \mid \operatorname{gcd}(\zeta \delta, e)$. Conversely, if (6.9) or one of the cases of (6.10) holds for each such $q$, then it is clear from the formula for $A(d i)$ in each case that $A(d i)$ represents all residue classes mod $q$ as $i$ runs through any complete set of residues mod $q$. It then follows from the Chinese Remainder Theorem that (6.8) holds.

We extract the following information from the proof of Proposition 6.9 .

Corollary 6.10. If (6.9) holds then $A(d i) \equiv u d i$.

If $(6.10)(i)$ holds then

$$
A(d i) \equiv \frac{v z d i}{k-1}
$$

If (6.10)(ii) holds then

$$
A(d i) \equiv \frac{z k}{k-1}(\mu u-v) d i .
$$

Hence if $\Gamma^{*} \in \mathcal{F}_{h}$ then, for each $q \mid \operatorname{gcd}(\zeta \delta, e)$,

$$
q|A(d i) \Leftrightarrow q| i \text {. }
$$

Proposition 6.11. Suppose that (5.3) and conditions (i) and (ii) of Lemma 5.6 hold. Then condition (iii) of Lemma 5.6 is equivalent to the two further conditions

$$
\begin{gathered}
\text { if } q \in S_{h}^{+} \cup T \text { and } t \equiv \kappa k^{-1} \equiv 1 \text { then } v \equiv 0 ; \\
\text { if } q \in S_{h}^{-} \cup T \text { and } t \equiv \kappa \text { then } v \equiv \mu u .
\end{gathered}
$$

Moreover, we then have

$$
A(d i) \equiv 0 \quad(\bmod q) \text { when } q \mid \gamma \text { and } i \equiv 0 \quad(\bmod \operatorname{gcd}(\delta, e)) .
$$

Proof. By Hypothesis 5.3, we have $\zeta \delta=d i_{0}$ where $i_{0}=\operatorname{gcd}(\zeta \delta, e)$. It then follows from $(6.4),(6.5),(6.9)$ and $(6.10)$ that $t^{d i} \equiv 1(\bmod e)$ whenever $i_{0} \mid i$.

By Lemma 4.3, condition (iii) of Lemma 5.6 is equivalent to the following two congruences:

$$
\begin{gathered}
A(\zeta \delta) \equiv 0 \quad(\bmod e) ; \\
v S(t, \zeta \delta) \equiv 0 \quad(\bmod g) .
\end{gathered}
$$

We will show that these are equivalent to (6.11) and (6.12). 
We first check that (6.14) and (6.15) give no conditions for primes $q \mid \operatorname{gcd}(\zeta \delta, e)=i_{0}$. We have $A\left(d i_{0}\right) \equiv 0$ by Corollary 6.10 , so $(6.14)$ holds. If also $q \mid g$, then by (6.10) either $t \equiv 1$, so that $S(t, \zeta \delta)=\zeta \delta \equiv$ 0 , or $t k \equiv 1$, so that $S(t, \zeta \delta) \equiv 0$ since $t^{\zeta \delta} \equiv 1$. Hence (6.15) holds.

Next suppose that $q \mid \operatorname{gcd}(\gamma, z)$. Then (6.15) gives no condition at q. As (6.14) is a special case of (6.13), we just need to verify (6.13) for these $q$. Now $t \equiv \kappa$ by (6.4), and also $k \equiv 1 \not \equiv \kappa$. In this case,

$$
A(d i)=u S(t k, d i) \equiv 0 \text { for all } i,
$$

giving (6.13).

Finally, let $q \mid \operatorname{gcd}(\gamma, g)$. Recall that

$$
A(d i)=u S(t k, d i)+v z k T(k, t, d i) .
$$

If $t \not \equiv 1 \not \equiv t k$ then $S(t k, \zeta \delta) \equiv T(k, t, \zeta \delta) \equiv S(t k, \zeta \delta) \equiv 0$, so (6.14), (6.15) hold with no conditions on $u, v$. Similarly, if $i_{0} \mid i$ then (6.13) holds. It remains to consider the special cases $t \equiv 1$ and $t k \equiv 1$.

If $t \equiv 1$, we cannot have $t \equiv \kappa$ since $\kappa \not \equiv 1$, but

$$
t k \equiv \kappa \Leftrightarrow k \equiv \kappa \Leftrightarrow q \in S_{h}^{+} \cup T .
$$

In this case (6.15) is equivalent to $v \equiv 0$, and then (6.14) holds for arbitrary $u$. This gives (6.11), and (6.13) also holds.

If $t k \equiv 1$, we cannot have $t k \equiv \kappa$, but

$$
t \equiv \kappa \Leftrightarrow k \equiv \kappa^{-1} \Leftrightarrow q \in S_{h}^{-} \cup T .
$$

As $t \not \equiv 1$, (6.15) holds for arbitrary $v$, and (6.14) becomes

$$
u \zeta \delta+z v \frac{k \zeta \delta}{k(t-1)} \equiv 0,
$$

which simplifies to (6.12) since $t \equiv k^{-1}$. Again, (6.13) holds.

Lemma 6.12. A quintuple $(t, a, c, u, v) \in \mathbb{Z}_{e}^{\times} \times \mathbb{Z}_{e} \times \mathbb{Z}_{g} \times \mathbb{Z}_{e} \times \mathbb{Z}_{g}$ belongs to $\mathcal{N}_{h}$ if and only if, for each prime $q \mid e$, its entries satisfy the conditions $\bmod q$ shown in Table 1, where $\lambda, \mu$ are defined in Propositions 6.8, 6.9 respectively.

Remark 6.13. Before proving Lemma 6.12, we make some comments about how to read Table 1. All congruences are modulo the relevant prime $q$. Entries shown as "arb." may be chosen arbitrarily mod $q$. Where there are two rows for a particular set of primes, $(t, a, u, c, v)$ may satisfy the conditions in either row. For example, if $q \in S_{h}^{+}$then either $t \equiv 1, a \not \equiv 0, c \equiv v \equiv 0$ or $t \equiv \kappa, a \not \equiv 0, c \equiv \lambda a$. The cells for $c, v$ when $q \mid z$ are empty since $c, v \in \mathbb{Z}_{g}$ so are not defined $\bmod q$. The final column of Table 1 will be explained in the proof of Lemma 7.1 below. 


\begin{tabular}{|c|c|c|c|c|c|c|}
\hline Primes $q$ & $t$ & $a$ & $u$ & $c$ & $v$ & Number \\
\hline$q \in P$ & $\kappa$ & $\not \equiv 0$ & arb. & & & $q(q-1)$ \\
\hline$q \in Q$ & 1 & 0 & $\equiv \equiv 0$ & & & $q-1$ \\
\hline$q \in R \cup S_{h}^{\prime}$ & $\kappa$ & $\not \equiv 0$ & arb. & $\lambda a$ & arb. & $2 q^{2}(q-1)$ \\
& $\kappa k^{-1}$ & $\neq \equiv 0$ & arb. & 0 & arb. & \\
\hline$q \in S_{h}^{+}$ & $\kappa k^{-1} \equiv 1$ & $\not \equiv 0$ & arb. & 0 & 0 & $q\left(q^{2}-1\right)$ \\
& $\kappa$ & $\not \equiv 0$ & arb. & $\lambda a$ & arb. & \\
\hline$q \in S_{h}^{-}$ & $\kappa$ & $\not \equiv 0$ & arb. & $\lambda a$ & $\mu u$ & $q\left(q^{2}-1\right)$ \\
& $\kappa k^{-1} \equiv \kappa^{2}$ & $\not \equiv 0$ & arb. & 0 & arb. & \\
\hline$q \in T$ & $\kappa \equiv-1$ & $\not \equiv 0$ & arb. & $\lambda a$ & $\mu u$ & $2 q(q-1)$ \\
& $\kappa k^{-1} \equiv 1$ & $\neq \equiv 0$ & arb. & 0 & 0 & \\
\hline$q \in U$ & 1 & 0 & arb. & 0 & $\not \equiv 0$ & $2 q(q-1)$ \\
& $k^{-1}$ & 0 & arb. & 0 & $\neq \equiv \mu u$ & \\
\hline
\end{tabular}

TABLE 1. Conditions for membership of $\mathcal{N}_{h}$.

Proof of Lemma 6.12. By Lemma 5.6 and Propositions 6.7, 6.8, 6.9 and $6.11,(t, a, c, u, v) \in \mathcal{N}_{h}$ if and only if the relevant conditions from (6.1)-(6.5) and (6.9)-(6.12) hold for each prime $q \mid e$. We pick out these conditions for each prime.

For $q \in P$, we have $q \mid \operatorname{gcd}(\gamma, z)$, so $a \not \equiv 0$ by $(6.1)$ and $t \equiv \kappa$ by (6.4).

For $q \in Q$, we have $q \mid \operatorname{gcd}(\zeta \delta, z)$, so $a \equiv 0$ by $(6.2)$ and $t \equiv 1, u \not \equiv 0$ by $(6.9)$.

If $q \in R$ then $\kappa \not \equiv k^{ \pm 1}$ since $\kappa$ has order $\rho_{q}$ and $k^{ \pm 1}$ has order $r_{q} \neq \rho_{q}$. If $q \in S_{h}^{\prime}$ then again $\kappa \not \equiv k^{ \pm 1}$. In both cases, $q \mid \operatorname{gcd}(\gamma, g)$. Thus by (6.5) either $t \equiv \kappa, c \equiv \lambda a$ or $t \equiv \kappa k^{-1}, c \equiv 0$. Moreover, $a \not \equiv 0$ by (6.1), and there are no restrictions on $u$ and $v$.

For $q \in S_{h}^{+}$, we have $q \mid \operatorname{gcd}(\gamma, g)$ and $\kappa \equiv k \not \equiv \pm 1$. Again, $a \not \equiv 0$ by (6.1), and by (6.5) either $t \equiv \kappa, c \equiv \lambda a$ or $t \equiv \kappa k^{-1} \equiv 1, c \equiv 0$. If $t \equiv 1$ then $v \equiv 0$ by (6.11), whereas if $t \equiv \kappa$ there is no restriction on $u$ and $v$.

For $q \in S_{h}^{-}$, we have $q \mid \operatorname{gcd}(\gamma, g)$ and $\kappa \equiv k^{-1} \not \equiv \pm 1$. As before, $a \not \equiv 0$ and either $t \equiv \kappa, c \equiv \lambda a$ or $t \equiv \kappa k^{-1}, c \equiv 0$. If $t \equiv \kappa$ then $\kappa k \equiv 1$, and by (6.12) we have $v=\mu u$, whereas if $t \equiv \kappa k^{-1}$ there is no restriction on $u$ and $v$.

For $q \in T$, we have $q \mid \operatorname{gcd}(\gamma, g)$ and $\kappa \equiv k \equiv-1 \not \equiv 1$. Again $a \not \equiv 0$ and either $t \equiv \kappa \equiv-1, c \equiv \lambda a$ or $t \equiv \kappa k^{-1} \equiv 1, c \equiv 0$. If $t \equiv 1$ then $v \equiv 0$ by (6.11), while if $t \equiv-1$ then $v \equiv \mu u$ by (6.12).

For $q \in U$ we have $q \mid \operatorname{gcd}(\zeta \delta, g)$. Then $a \equiv 0$ and $c \equiv 0$ by (6.2) and (6.3). Moreover, by (6.10), either $t \equiv 1, v \not \equiv 0$ or $t \equiv k^{-1}, v \not \equiv \mu u$. 


\section{Counting Hopf-Galois Structures}

In this section, we complete the proof of Theorem 2.2.

Lemma 7.1. Suppose that Hypothesis 5.3 holds, and let $1 \leq h \leq w$. Then

$$
\left|\mathcal{N}_{h}\right|=\varphi(e) 2^{\omega(g)} g \gamma\left(\prod_{q \in T} \frac{1}{q}\right)\left(\prod_{q \in S_{h}} \frac{q+1}{2 q}\right) .
$$

In particular, $\mathcal{F}_{h}$ is not empty.

Proof. By Lemma 6.12, membership of $\mathcal{N}_{h}$ is determined by conditions $\bmod q$ for each prime $q \mid e$ separately, so, by the Chinese Remainder Theorem, the number of possible quintuples in $\mathcal{N}_{h}$ is the product over all $q$ of the number of possible quintuples $\bmod q$.

The final column of Table 1 above shows the number of possibilities for $(t, a, c, u, v) \bmod q$ for each prime $q$. (If $q \mid z$, we ignore $c, v$ as they are only defined $\bmod g$.) For example, if $q \in S_{h}^{+}$then either $t \equiv 1$ or $t \equiv \kappa$ (where, as before, $\kappa$ really means $\kappa_{h}$ ). If $t \equiv 1$, we have $a \not \equiv 0, u$ arbitrary, but $c \equiv v \equiv 0$, giving $(q-1) q$ quintuples mod $q$. If $t \equiv \kappa$ then again $a \not \equiv 0, c \equiv \lambda a$, and now $u, v$ are arbitrary. This gives a further $(q-1) q^{2}$ quintuples. Thus the total number of quintuples $\bmod q$ is $(q-1) q+(q-1) q^{2}=q\left(q^{2}-1\right)$, as shown in Table 1 . The other entries in the final column of Table 1 are obtained by similar (but usually simpler) calculations, which we leave to the reader.

Noting that $\prod_{q \mid e}(q-1)=\varphi(e)$, we therefore have

$$
\begin{aligned}
\left|\mathcal{N}_{h}\right| & =\varphi(e)\left(\prod_{q \in P} q\right)\left(\prod_{q \in R \cup S_{h}^{\prime}} 2 q^{2}\right)\left(\prod_{q \in S_{h}} q(q+1)\right)\left(\prod_{q \in T \cup U} 2 q\right) \\
& =\varphi(e)\left(\prod_{q \in P} q\right)\left(\prod_{q \in R \cup S \cup T} 2 q^{2}\right)\left(\prod_{q \in S_{h}} \frac{q+1}{2 q}\right)\left(\prod_{q \in T} \frac{1}{q}\right)\left(\prod_{q \in U} 2 q\right) \\
& =\varphi(e)\left(\prod_{q \in R \cup S \cup T \cup U} 2 q\right)\left(\prod_{q \in P \cup R \cup S \cup T} q\right)\left(\prod_{q \in T} \frac{1}{q}\right)\left(\prod_{q \in S_{h}} \frac{q+1}{2 q}\right) \\
& =\varphi(e)\left(\prod_{q \mid g} 2 q\right)\left(\prod_{q \mid \gamma} q\right)\left(\prod_{q \in T} \frac{1}{q}\right)\left(\prod_{q \in S_{h}} \frac{q+1}{2 q}\right) \\
& =\varphi(e)\left(2^{\omega(g)} g\right) \gamma\left(\prod_{q \in T} \frac{1}{q}\right)\left(\prod_{q \in S_{h}} \frac{q+1}{2 q}\right) .
\end{aligned}
$$


Proof of Theorem 2.2. Given the two groups $G, \Gamma$ of squarefree order $n$, we want to determine the number $e(\Gamma, G)$ of Hopf-Galois structures of type $G$ on a Galois extension with Galois group isomorphic to $\Gamma$. This is related by the formula (3.1) to the number $e^{\prime}(G, \Gamma)$ of regular subgroups in $\operatorname{Hol}(G)$ isomorphic to $\Gamma$.

If $\gamma \nmid e$ then by Proposition 5.2 there are no such regular subgroups and hence no Hopf-Galois structures.

If $\gamma \mid e$, so Hypothesis 5.3 holds, then by (5.4) and Lemma 7.1 we have

$$
e^{\prime}(G, \Gamma)=\frac{\varphi(\delta) 2^{\omega(g)} g}{w}\left(\prod_{q \in T} \frac{1}{q}\right) \sum_{h=1}^{w}\left(\prod_{q \in S_{h}} \frac{q+1}{2 q}\right) .
$$

Since $|\operatorname{Aut}(G)|=g \varphi(e)$ by Lemma 4.1, and similarly $|\operatorname{Aut}(\Gamma)|=\gamma \varphi(\epsilon)$, it follows from (3.1) that

$$
\begin{aligned}
e(\Gamma, G) & =\frac{|\operatorname{Aut}(\Gamma)|}{|\operatorname{Aut}(G)|} e^{\prime}(G, \Gamma) \\
& =\frac{\gamma \varphi(\epsilon) \varphi(\delta) 2^{\omega(g)} g}{g \varphi(e) w}\left(\prod_{q \in T} \frac{1}{q}\right) \sum_{h=1}^{w}\left(\prod_{q \in S_{h}} \frac{q+1}{2 q}\right) .
\end{aligned}
$$

As $\varphi(\epsilon) \varphi(\delta)=\varphi(n)=\varphi(e) \varphi(d)$, we therefore have

$$
e(\Gamma, G)=\frac{\gamma \varphi(d) 2^{\omega(g)}}{w}\left(\prod_{q \in T} \frac{1}{q}\right) \sum_{h=1}^{w}\left(\prod_{q \in S_{h}} \frac{q+1}{2 q}\right) .
$$

This completes the proof of Theorem 2.2.

\section{AN EXAMPLE WHERE $n$ HAS 7 PRIME FACTORS}

We consider some of the groups of order

$$
n=2 \cdot 3 \cdot 7 \cdot 43 \cdot 127 \cdot 211 \cdot 337=16309243734,
$$

a squarefree integer with 7 prime factors. The numerical calculations in this section were performed using MAPLE.

Using a formula of Hölder [Hö195], recalled as [AB18, eqn. (1)], we find that there are 272736 isomorphism classes of groups of order $n$. We consider just four of these, all of which have $g=43 \cdot 127 \cdot 211 \cdot 337$.

We first choose $a_{1} \in \mathbb{Z}_{43}^{\times}, a_{2} \in \mathbb{Z}_{127}^{\times}, a_{3} \in \mathbb{Z}_{211}^{\times}, a_{4} \in \mathbb{Z}_{337}^{\times}$all having order 42. (For example, we may take $a_{1}=3, a_{2}=5, a_{3}=26, a_{4}=21$.) We then specify our groups $G$ by stipulating that $k$ is congruent to a certain power of $a_{1}\left(\right.$ resp. $\left.a_{2}, a_{3}, a_{4}\right) \bmod 43($ resp. 127, 211, 377) as shown in Table 2.

We also show in Table 2 the value of $r_{q}$ for each of the primes $q$ dividing $g$. In each case, $d$ is the least common multiple of these values. 


\begin{tabular}{|c|c|c|c|c|c|c|c|c|c|}
\hline & $k \bmod 43$ & $k \bmod 127$ & $k \bmod 211$ & $k \bmod 337$ & $r_{43}$ & $r_{127}$ & $r_{211}$ & $r_{337}$ & $d$ \\
\hline$G_{1}$ & $a_{1}^{21}$ & $a_{2}^{14}$ & $a_{3}^{6}$ & $a_{4}^{2}$ & 2 & 3 & 7 & 21 & 42 \\
\hline$G_{2}$ & $a_{1}^{21}$ & $a_{2}^{14}$ & $a_{3}^{12}$ & $a_{4}^{2}$ & 2 & 3 & 7 & 21 & 42 \\
\hline$G_{3}$ & $a_{1}$ & $a_{2}^{2}$ & $a_{3}^{3}$ & $a_{4}^{6}$ & 42 & 21 & 14 & 7 & 42 \\
\hline$G_{4}$ & $a_{1}^{21}$ & $a_{2}^{6}$ & $a_{3}^{6}$ & $a_{4}^{3}$ & 2 & 7 & 7 & 14 & 14 \\
\hline
\end{tabular}

TABLE 2. Parameters for some groups of order 16309243734.

For $G_{1}, G_{2}, G_{3}$ we have $d=42$ so $z=1$ and $e=g$. For $G_{4}$, we assume further that $k \equiv 1(\bmod 3)$, so that $d=14$ and $z=3$ and $e=3 g$.

Remark 8.1. No two of our groups $G_{i}$ are isomorphic, since no two of our choices of $k$ generate the same cyclic subgroup of $\mathbb{Z}_{e}^{\times}$. Nevertheless, $G_{1}$ and $G_{2}$ have the same values of $d, g, z$ and $r_{q}$ for all $q \mid e$, showing that these parameters are not in general sufficient to determine the isomorphism class of $G$. Moreover, $d, g$ and $z$ are not in general sufficient to determine the $r_{q}$, as shown by $G_{2}$ and $G_{3}$.

We now fix $\Gamma=G_{1}$, without loss of generality taking $\kappa$ to be the value of $k$ specified for $G_{1}$. We will use Theorem 2.2 to determine $e\left(\Gamma, G_{i}\right)$ for $i=1, \ldots, 4$.

8.1. $e\left(\Gamma, G_{1}\right)$ : Since $d=\delta$, the group $\Delta$ is trivial and $w=\varphi(d)=12$. The set $\mathcal{K}$ consists of the 12 elements $\kappa, \kappa^{5}, \kappa^{11}, \kappa^{13}, \kappa^{17}, \kappa^{19}, \kappa^{23}$, $\kappa^{25}, \kappa^{29}, \kappa^{31}, \kappa^{37}, \kappa^{41}$. We label these as $\kappa_{1}, \ldots, \kappa_{12}$ in the order listed. Then the $\kappa_{h}$ are chosen as in Remark 5.4.

We have $R=\emptyset, S=\{127,211,337\}, T=\{43\}$. We determine the sets $S_{h}^{+}$by considering each prime $q \in S$. For $q=127$, we have $r_{q}=3$ so $k \equiv \kappa_{h}$ if and only if $\kappa_{h} \equiv \kappa^{r}$ with $r \equiv 1(\bmod 3)$. This occurs for $h=1,4,6,8,10,11$. For $q=211$, we have $r_{q}=7$ and $k \equiv \kappa_{h}$ for $h=1,9$. For $q=337$, we have $r_{q}=21$ and $k \equiv \kappa_{h}$ only for $h=1$. Thus

$$
S_{h}^{+}= \begin{cases}\{127,211,337\} & \text { if } h=1, \\ \{127\} & \text { if } h=4,6,8,10,11 \\ \{211\} & \text { if } h=9, \\ \emptyset & \text { if } h=2,3,5,7,12\end{cases}
$$

For each $q \in S$, we have $q \in S_{h}^{+}$for $w / \varphi\left(r_{q}\right)$ values of $h$, as explained in Proposition 6.6. Since $S_{h}^{-}=S_{w+1-h}^{+}$, the sets $S_{h}=S_{h}^{+} \cup S_{h}^{-}$are as 
follows:

$$
S_{h}= \begin{cases}\{127,211,337\} & \text { if } h=1,12 \\ \{127\} & \text { if } h=2,3,5,6,7,8,10,11 \\ \{127,211\} & \text { if } h=4,9 .\end{cases}
$$

Then the number $e\left(\Gamma, G_{1}\right)=e(\Gamma, \Gamma)$ of Hopf-Galois structures is

$$
\begin{aligned}
e\left(\Gamma, G_{1}\right) & =\frac{2^{\omega(g)} \varphi(d) \gamma}{w}\left(\prod_{q \in T} \frac{1}{q}\right) \sum_{h=1}^{w} \prod_{q \in S_{h}} \frac{q+1}{2 q} \\
& =\frac{2^{4} \varphi(42) \cdot 43 \cdot 127 \cdot 211 \cdot 337}{12} \cdot \frac{1}{43} \cdot \\
& \left(2 \times \frac{128}{254} \times \frac{212}{422} \times \frac{338}{674}+8 \times \frac{128}{254}+2 \times \frac{128}{254} \times \frac{212}{422}\right) \\
& =692355072 .
\end{aligned}
$$

8.2. $e\left(\Gamma, G_{2}\right)$ : We now have $211 \in S_{h}$ if and only if $\kappa_{h} \equiv \kappa^{r}$ with $r \equiv \pm 2(\bmod 7)$, which occurs for $h=2,6,7,11$. Thus

$$
S_{h}= \begin{cases}\{127,337\} & \text { if } h=1,12 \\ \{127,211\} & \text { if } h=2,6,7,11 \\ \{127\} & \text { if } h=3,4,5,8,9,10\end{cases}
$$

The sets $S_{h}$ in this case are different from those in $\S 8.1$, even though the $r_{q}$ have not changed. The number of Hopf-Galois structures is now

$$
\begin{aligned}
e\left(\Gamma, G_{2}\right)= & \frac{2^{4} \varphi(42) \cdot 43 \cdot 127 \cdot 211 \cdot 337}{12} \cdot \frac{1}{43} . \\
& \left(2 \times \frac{128}{254} \times \frac{338}{674}+4 \times \frac{128}{254} \times \frac{212}{422}+6 \times \frac{128}{254}\right) \\
= & 656228352 .
\end{aligned}
$$

8.3. $e\left(\Gamma, G_{3}\right)$ : This time there are no primes $q \mid e$ with $r_{q}=\rho_{q}$, so $S=T=\emptyset$ and $R=\{43,127,211,337\}$. The number of Hopf-Galois structures is now

$$
\begin{aligned}
e\left(\Gamma, G_{3}\right) & =\frac{2^{4} \varphi(42) \cdot 43 \cdot 127 \cdot 211 \cdot 337}{12} \cdot \sum_{h=1}^{12} 1 \\
& =74556542784 .
\end{aligned}
$$


8.4. $e\left(\Gamma, G_{4}\right)$ : In the previous cases, we had $w=|\mathcal{K}|$, so that the orbits of $\Delta$ on $\mathcal{K}$ were singletons. In our final case, this does not hold. Indeed, we have $w=\varphi(\operatorname{gcd}(14,42))=6$, and a system of orbit representatives of $\mathcal{K}$ under $\Delta=\{1,29\}$, chosen as in Remark 5.4, is $\kappa_{1}=\kappa, \kappa_{2}=\kappa^{5}$, $\kappa_{3}=\kappa^{11}, \kappa_{4}=\kappa^{31}, \kappa_{5}=\kappa^{37}, \kappa_{6}=\kappa^{41}$.

We find $R=\{127,337\}, S=\{211\}, T=\{43\}$. As $k \equiv \kappa(\bmod 211)$ and $r_{221}=7$, we have $211 \in S_{h}^{+}$only for $h=1$ and $211 \in S_{h}^{-}$only for $h=6$. The number of Hopf-Galois structures is now

$$
\begin{aligned}
e\left(\Gamma, G_{4}\right) & =\frac{2^{4} \varphi(14) \cdot 43 \cdot 127 \cdot 211 \cdot 337}{6} \cdot \frac{1}{43} . \\
& =723131904 .
\end{aligned}
$$

\section{Special CASES}

In this section, we give some corollaries of our main result Theorem 2.2 .

9.1. When $\Gamma$ or $G$ is cyclic or dihedral. We begin by reproving [AB18, Theorem 1]

Corollary 9.1. Let $G=G(d, e, k)$ be an arbitrary group of squarefree order $n$. Then a cyclic extension of degree $n$ admits precisely $2^{\omega(g)} \varphi(d)$ Hopf-Galois structures of type $G$, where $g=e / \operatorname{gcd}(k-1, e)$.

Proof. For $\Gamma$ cyclic, we have $\gamma=\delta=1$, so $w=1$ and the sets $R, S, T$ are empty. Then Theorem 2.2 gives

$$
e(\Gamma, G)=2^{\omega(g)} \varphi(d) .
$$

Corollary 9.2. Let $\Gamma=G(\delta, \epsilon, \kappa)$ be an arbitrary group of squarefree order $n$. A Galois extension with group $\Gamma$ admits precisely $\gamma=$ $\epsilon / \operatorname{gcd}(\kappa-1, \epsilon)$ Hopf-Galois structures of cyclic type.

Proof. For $G$ cyclic, we have $d=g=1$ so again $w=1$ and $R, S, T$ are empty. Thus $e(\Gamma, G)=\gamma$.

Corollary 9.3. Let $m$ be an odd squarefree integer, let $L / K$ be a Galois extension of degree $n=2 m$ with $\operatorname{Gal}(L / K) \cong D_{2 m}$, the dihedral group of order $n$. Let $G=G(d, e, k)$ be an arbitrary group of order $n$. Then, 
with $g=e / \operatorname{gcd}(k-1, e)$, the number of Hopf-Galois structures of type $G$ on $L / K$ is

$$
e\left(D_{2 m}, G\right)= \begin{cases}m & \text { if } d=1, \\ \frac{2^{\omega(g)} m}{g} & \text { if } d=2, \\ 0 & \text { otherwise. }\end{cases}
$$

Proof. Taking $\Gamma=D_{2 m}$, we have $\delta=2, \gamma=m, \zeta=1, w=1$ and $\rho_{q}=2$ for all primes $q \mid m$. If $d=1$ the result follows from Corollary 9.2. If $d>2$ then $\gamma \nmid e$, so $e\left(D_{2 m}, G\right)=0$. If $d=2$, we have $r_{q}=2$ for each prime $q \mid g$, so $T=\{q \mid g\}$ and $R, S$ are empty. The stated formula follows from Theorem 2.2 .

Corollary 9.4. Let $m$ be an odd squarefree integer, and let $\Gamma=G(\delta, \epsilon, \kappa)$ be an arbitrary group of order $n=2 \mathrm{~m}$. Then the number of Hopf-Galois structures of dihedral type on a Galois extension with Galois group $\Gamma$ is

$$
e\left(\Gamma, D_{2 m}\right)=2^{\omega(m)} \gamma\left(\prod_{q \mid \gamma, \rho_{q}=2} \frac{1}{q}\right) .
$$

Proof. We let $G=D_{2 m}$, so $d=2, g=m, z=1$ and $w=1$. Since $2 \nmid \gamma$ (see Remark 6.1) we necessarily have $\gamma \mid g$. Then $r_{q}=2$ for all $q \mid m$, so $R$ and $S$ are empty and $T=\left\{q \mid \gamma: \rho_{q}=2\right\}$. The stated formula then follows from Theorem 2.2.

9.2. When $n$ is the product of two primes. We next recover the results of [Byo04] which count Hopf-Galois structures on Galois extensions of degree $n=p q$, where $p>q$ are primes. If $p \not \equiv 1(\bmod q)$ then any group of order $n$ is cyclic, and it follows from Corollary 9.1 (with $d=k=1$ ) that any Galois extension of degree $n$ admits only the classical Hopf-Galois structure. This was already proved in [Byo96]. We therefore assume that $p \equiv 1(\bmod q)$. We then have two groups of order $n$, the cyclic group $C_{n}$ (for which $g=d=1$ and $z=p q$ ) and the nonabelian group $C_{p} \rtimes C_{q}$ (for which $g=p, d=q, z=1$ ).

Corollary 9.5. Let $n=p q$, where $p$, $q$ are primes with $p \equiv 1(\bmod q)$. Let $\Gamma, G$ be groups of order pq. Then the number of Hopf-Galois structures of type $G$ on a Galois extension with Galois group $\Gamma$ is as given in Table 3.

Proof. If either $G$ or $\Gamma$ is cyclic, the result follows from Corollary 9.1 or Corollary 9.2. If $G=\Gamma=C_{p} \rtimes C_{q}$ with $q=2$ (so $G$ and $\Gamma$ are both dihedral), the result follows from Corollary 9.3, or, alternatively, from 


\begin{tabular}{|c|c|c|}
\hline & $G=C_{n}$ & $G=C_{p} \rtimes C_{q}$ \\
\hline$\Gamma=C_{n}$ & 1 & $2(q-1)$ \\
\hline$\Gamma=C_{p} \rtimes C_{q}$ & $p$ & $2 p(q-2)+2$ \\
\hline
\end{tabular}

TABLE 3. Hopf-Galois structures for two primes.

Corollary 9.4. It remains to consider the case $G=\Gamma=C_{p} \rtimes C_{q}$ with $q>2$. Then $d=\delta=q$ so $w=\varphi(q)=q-1$. Thus $R$ and $T$ are empty and $S=\{p\}$ with $r_{p}=\rho_{p}=q-1$. There is one value of $h$ with $p \in S_{h}^{+}$ and one with $p \in S_{h}^{-}$. We therefore have

$$
e(\Gamma, G)=2 p\left(2 \times \frac{p+1}{2 p}+(q-3)\right)=2 p(q-2)+2 .
$$

9.3. When $n$ is the product of three primes. In this final subsection, we consider the case where $n$ is the product of three distinct primes $p_{1}<p_{2}<p_{3}$. This extends work of Kohl [Koh13, Koh16]. In [Koh13], Kohl takes $p_{1}=2$ and $p_{3}=2 p_{2}+1$, so that $q=p_{2}>2$ is a Sophie Germain prime and $p=p_{3}$ is a safeprime. He calculates $e(\Gamma, G)$ for all possible pairs $\Gamma, G$. The results for $\Gamma=C_{p} \rtimes C_{p-1}=\operatorname{Hol}\left(C_{p}\right)$ were already obtained in [Chi03]. In [Koh16], Kohl handles the case $p_{1}>2$, with $p_{3} \equiv p_{2} \equiv 1\left(\bmod p_{1}\right)$ but $p_{3} \not \equiv 1\left(\bmod p_{2}\right)$.

We first describe the groups of order $n=p_{1} p_{2} p_{3}$. Subject to certain congruence conditions, there are 6 possible factorisations $n=d g z$ which give rise to groups of order $n$. For ease of reference, we label these factorisations $1-6$ as shown in Table 4 . The last column shows the number of isomorphism types of group $G$ for each factorisation.

In all cases except Factorisation 4 , the group $\mathbb{Z}_{g}^{\times}$is cyclic and therefore has a unique subgroup of order $d$, so that there is a unique isomorphism type of group for the given factorisation. For Factorisation 4 , however, the Sylow $p_{1}$-subgroup of $\mathbb{Z}_{g}^{\times}=\mathbb{Z}_{p_{2}}^{\times} \times \mathbb{Z}_{p_{3}}^{\times}$is of order $p_{1}^{2}$ and contains $p_{1}+1$ subgroups of order $d=p_{1}$, of which $p_{1}-1$ project nontrivally to both factors. In this case, we obtain $p_{1}-1$ distinct isomorphism classes. Thus there are in total $p_{1}+4$ isomorphism classes of groups of order $n$, provided that all the indicated congruence conditions hold.

We examine more carefully the $p_{1}-1$ groups from Factorisation 4 when $p_{1}>2$. We may assume that the corresponding values of $k$ are all congruent $\bmod p_{2}$, but run through all $p_{1}-1$ residue classes of order $p_{1} \bmod p_{3}$. Given one such group $G=G\left(p_{1}, p_{2} p_{3}, k\right)$, we write 


\begin{tabular}{|c|ccc|c|c|}
\hline Factorisation & $d$ & $g$ & $z$ & Condition & \# groups \\
\hline 1 & 1 & 1 & $p_{1} p_{2} p_{3}$ & & 1 \\
2 & $p_{1}$ & $p_{2}$ & $p_{3}$ & $p_{2} \equiv 1\left(\bmod p_{1}\right)$ & 1 \\
3 & $p_{1}$ & $p_{3}$ & $p_{2}$ & $p_{3} \equiv 1\left(\bmod p_{1}\right)$ & 1 \\
4 & $p_{1}$ & $p_{2} p_{3}$ & 1 & $p_{2} \equiv p_{3} \equiv 1\left(\bmod p_{1}\right)$ & $p_{1}-1$ \\
5 & $p_{2}$ & $p_{3}$ & $p_{1}$ & $p_{3} \equiv 1\left(\bmod p_{2}\right)$ & 1 \\
6 & $p_{1} p_{2}$ & $p_{3}$ & 1 & $p_{3} \equiv 1\left(\bmod p_{1} p_{2}\right)$ & 1 \\
\hline
\end{tabular}

TABLE 4. Isomorphism types for groups of order $n=p_{1} p_{2} p_{3}$.

$\widehat{G}=G\left(p_{1}, p_{2} p_{3}, \widehat{k}\right)$ where $\widehat{k} \equiv k\left(\bmod p_{2}\right)$ and $\widehat{k} \equiv k^{-1}\left(\bmod p_{3}\right)$. Thus the groups from Factorisation 4 come in pairs $G, \widehat{G}$.

We now determine $e(\Gamma, G)$ as the factorisations of $n$ corresponding to $G$ and $\Gamma$ each run through the 6 possibilities in Table 4 . For brevity, we state our result only in the situation where all the congruence conditions $p_{i} \equiv 1\left(\bmod p_{j}\right)$ for $i>j$ are satisfied. The results in the other cases are easily obtained from this, merely by omitting those $G$ and $\Gamma$ for which the relevant congruence conditions (as shown in Table 4) do not hold.

Theorem 9.6. Let $n=p_{1} p_{2} p_{3}$ where $p_{1}<p_{2}<p_{3}$ are primes and $p_{i} \equiv 1\left(\bmod p_{j}\right)$ for $i>j$. Let $G, \Gamma$ be groups of order $n$. Then $e(\Gamma, G)$, the number of Hopf-Galois structures of type $G$ on a Galois extension $L / K$ with $\operatorname{Gal}(L / K) \cong \Gamma$, is as shown in Table 5 if $p_{1}=2$, respectively Table 6 if $p_{1}>2$. The rows (respectively, columns) of these tables correspond to the factorisation of $n$ giving $\Gamma$ (respectively, $G)$, as in Table 4. In the case that $p_{1}>2$ and $G, \Gamma$ both come from Factorisation 4, we have

$$
e(\Gamma, G)= \begin{cases}4 p_{1} p_{2} p_{3}-10 p_{2} p_{3}+2 p_{2}+2 p_{3}+2 & \text { if } G \cong \Gamma \text { or } \widehat{\Gamma} \\ 4 p_{1} p_{2} p_{3}-12 p_{2} p_{3}+4 p_{2}+4 p_{3} & \text { if } G \approx \Gamma \text { or } \widehat{\Gamma}\end{cases}
$$

Proof. The entries shown as 0 in either table correspond to cases where $\gamma \nmid e$, so by Proposition 5.2 there are no regular subgroups in $\operatorname{Hol}(G)$ isomorphic to $\Gamma$ and $e(\Gamma, G)=0$.

We show in Table 7 the number $w$ and the sets $R, S, T$ for each combination of $G$ and $\Gamma$. For brevity, we only include the sets $R, S, T$ when they are nonempty, and we give these sets in the case $p_{1}=2$. If $p_{1} \neq 2$, we must replace $S$ by $S \cup T$ and $T$ by the empty set (cf. Remark 6.4). The four empty cells correspond to cases where Hypothesis 5.3 is not satisfied. 


\begin{tabular}{|c|c|c|c|c|c|c|}
\hline$\downarrow \Gamma \quad G \rightarrow$ & 1 & 2 & 3 & 4 & 5 & 6 \\
\hline 1 & 1 & 2 & 2 & 4 & $2\left(p_{2}-1\right)$ & $2\left(p_{2}-1\right)$ \\
\hline 2 & $p_{2}$ & 2 & $2 p_{2}$ & 4 & 0 & 0 \\
\hline 3 & $p_{3}$ & $2 p_{3}$ & 2 & 4 & $2\left(p_{2}-1\right) p_{3}$ & $2\left(p_{2}-1\right) p_{3}$ \\
\hline 4 & $p_{2} p_{3}$ & $2 p_{3}$ & $2 p_{2}$ & 4 & 0 & 0 \\
\hline 5 & $p_{3}$ & $2 p_{3}$ & $2 p_{3}$ & $4 p_{3}$ & $2+2\left(p_{2}-2\right) p_{3}$ & $2\left(p_{2}-1\right) p_{3}$ \\
\hline 6 & $p_{3}$ & $2 p_{3}$ & $2 p_{3}$ & $4 p_{3}$ & $2\left(p_{2}-1\right) p_{3}$ & $2+2\left(p_{2}-2\right) p_{3}$ \\
\hline
\end{tabular}

TABLE 5. Numbers of Hopf-Galois structures for $n=$ $p_{1} p_{2} p_{3}$ with $p_{1}=2$.

Except in the case $p_{1}>2$ and $g=\gamma=p_{2} p_{3}$ (that is, $G$ and $\Gamma$ both come from Factorisation 4), the set $S$ is either empty or consists of a single prime $p$. We have

$$
e(\Gamma, G)=2^{\omega(g)} \varphi(d) \gamma\left(\prod_{q \in T} \frac{1}{q}\right) \text { if } S=\emptyset .
$$

If $S=\{p\}$ then $p=p_{2}$ or $p_{3}$, and $r_{p}=d>2$. Then $p \in S_{h}^{+}$(respectively, $S_{h}^{-}$) for $w / \varphi(d)$ values of $h$, so the sum in the expression for the number $e(\Gamma, G)$ of Hopf-Galois structures simplifies to

$$
\begin{aligned}
\sum_{h=1}^{w} \prod_{q \in S_{h}} \frac{q+1}{2 q} & =\frac{2 w}{\varphi(d)} \cdot \frac{p+1}{2 p}+\left(w-\frac{2 w}{\varphi(d)}\right) \\
& =\frac{w}{\varphi(d) p}(1+[\varphi(d)-1] p) .
\end{aligned}
$$

Then we have

$$
e(\Gamma, G)=\frac{2^{\omega(g)} \gamma}{p}\left(\prod_{q \in T} \frac{1}{q}\right)(1+[\varphi(d)-1] p) \text { if } S=\{p\} .
$$

From Table 7, (9.2) and (9.3), we find that that when $p_{1}=2$ the values of $e(\Gamma, G)$ are as in Table 5 , and when $p_{1}>2$ (but $G$ and $\Gamma$ do not both come from Factorisation 4), they are as in Table 6.

We now examine the omitted case where $p_{1}>2$ and $G, \Gamma$ both come from Factorisation 4. We have $d=\delta=p_{1}$ and $w=p_{1}-1 \geq 2$. If $\kappa \equiv k$ or $\widehat{k}$ then $S_{h}=\left\{p_{2}, p_{3}\right\}$ for two of the $p_{1}-1$ possible values of $h$, and $S_{h}=\emptyset$ for the rest. This occurs for $G \cong \Gamma$ or $\widehat{\Gamma}$. Otherwise, there are two values of $h$ with $S_{h}=\left\{p_{2}\right\}$ and a further two with $S_{h}=\left\{p_{3}\right\}$. 

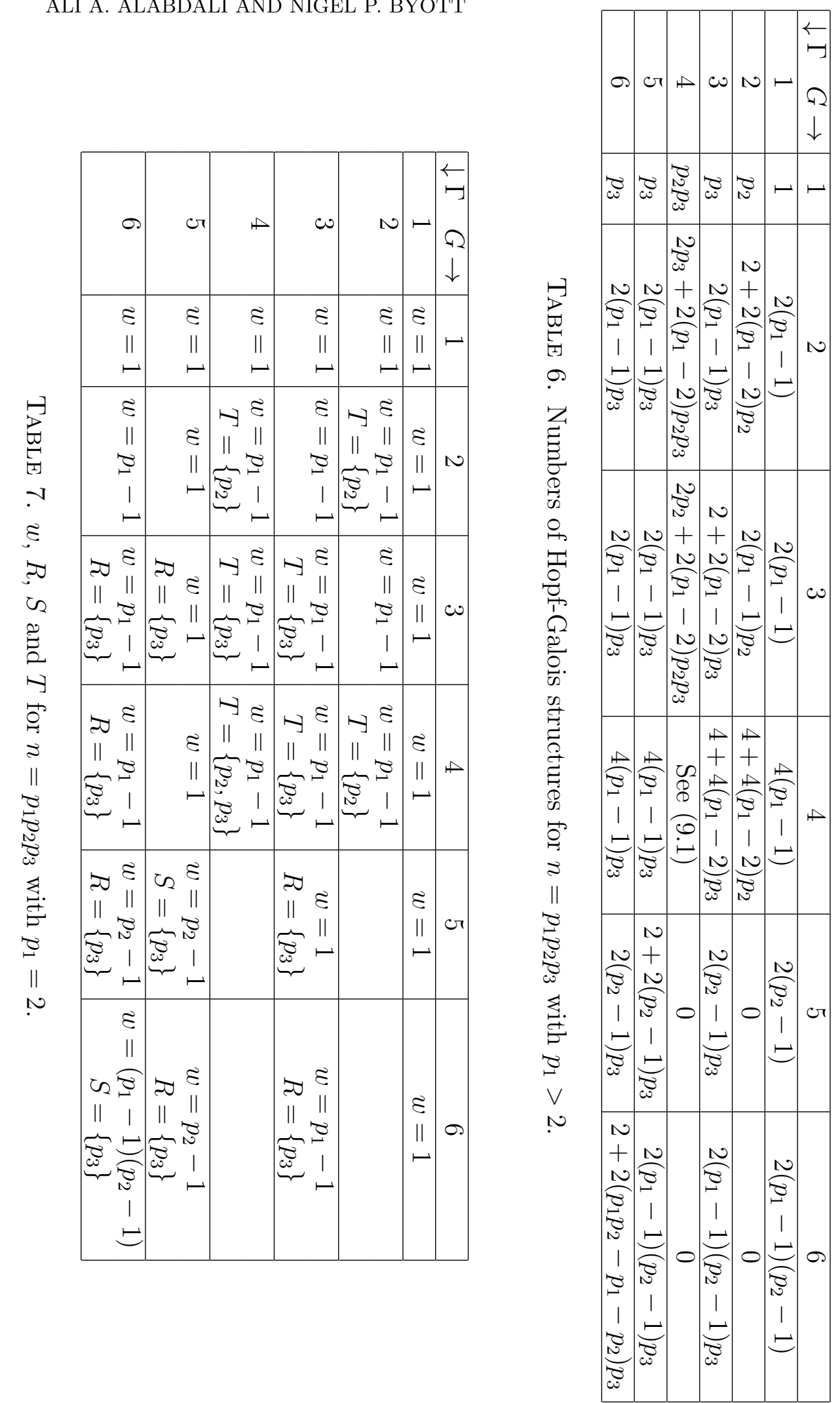
Thus, for $G \cong \Gamma$ or $\widehat{\Gamma}$, we have

$$
e(\Gamma, G)=\frac{2^{2}\left(p_{1}-1\right) p_{2} p_{3}}{p_{1}-1}\left(2 \cdot \frac{p_{2}+1}{2 p_{2}} \cdot \frac{p_{3}+1}{2 p_{3}}+\left(p_{1}-3\right)\right)
$$

while for $G ¥ \Gamma$ or $\widehat{\Gamma}$, we get

$$
e(\Gamma, G)=\frac{2^{2}\left(p_{1}-1\right) p_{2} p_{3}}{p_{1}-1}\left(2 \cdot \frac{p_{2}+1}{2 p_{2}}+2 \cdot \frac{p_{3}+1}{2 p_{3}}+\left(p_{1}-5\right)\right) .
$$

Hence we have (9.1).

Remark 9.7. Our results agree with those of [Koh13, Koh16]. When $p_{1}=2$ and $p_{3}=2 p_{2}+1$, the groups corresponding to the factorisations in Table 4 are denoted in [Koh13] as $C_{m p}, C_{p} \times D_{q}, C_{q} \times D_{p}, D_{p q}$, $F \times C_{2}$, Hol $C_{p}$, respectively. Our results in Table 5 in this case then agree with [Koh13, Theorem 5.1]. When $p_{1}>2$, with $p_{3} \equiv p_{2} \equiv 1$ $\left(\bmod p_{1}\right)$ but $p_{3} \not \equiv 1\left(\bmod p_{2}\right)$, only Factorisations $1-4$ in our Table 4 occur. In [Koh16], the corresponding groups are denoted respectively by $C_{p_{3} p_{2} p_{1}}, C_{p_{3}} \times\left(C_{p_{2}} \rtimes C_{p_{1}}\right), C_{p_{2}} \times\left(C_{p_{3}} \rtimes C_{p_{1}}\right), C_{p_{3} p_{2}} \rtimes C_{p_{1}}$, where the last case gives $p_{1}-1$ isomorphism classes of groups, coming from different actions of $C_{p_{1}}$ on $C_{p_{3} p_{2}}$. Our Table 6 (with the last two rows and columns omitted) matches the first table in [Koh16, Theorem 2.4], and the two cases in (9.1) respectively match the cases $j=i,-i$ and $j \neq i,-i$ in the second table there.

\section{REFERENCES}

[AB] Ali A. Alabdali and Nigel P. Byott, Skew braces of squarefree order, J. Algebra Appl., To appear.

[AB18] , Counting Hopf-Galois structures on cyclic field extensions of squarefree degree, J. Algebra 493 (2018), 1-19. MR 3715201

[Ala18] Ali A. Alabdali, Hopf-Galois structures on Galois extensions of fields of squarefree degree, Ph.D. thesis, University of Exeter, 2018, Available at https://ore.exeter.ac.uk/repository/handle/10871/33782.

[Bac16] David Bachiller, Counterexample to a conjecture about braces, J. Algebra 453 (2016), 160-176. MR 3465351

[Byo96] N. P. Byott, Uniqueness of Hopf Galois structure for separable field extensions, Comm. Algebra 24 (1996), no. 10, 3217-3228. MR 1402555

[Byo04] Nigel P. Byott, Hopf-Galois structures on Galois field extensions of degree pq, J. Pure Appl. Algebra 188 (2004), no. 1-3, 45-57. MR 2030805

[CC99] Scott Carnahan and Lindsay Childs, Counting Hopf Galois structures on non-abelian Galois field extensions, J. Algebra 218 (1999), no. 1, 81-92. MR 1704676

[Chi03] Lindsay N. Childs, On Hopf Galois structures and complete groups, New York J. Math. 9 (2003), 99-115. MR 2016184 
[CRV18] Teresa Crespo, Anna Rio, and Montserrat Vela, Hopf Galois structures on symmetric and alternating extensions, New York J. Math. 24 (2018), 451-457. MR 3855635

[CS69] Stephen U. Chase and Moss E. Sweedler, Hopf algebras and Galois theory, Lecture Notes in Mathematics, Vol. 97, Springer-Verlag, Berlin-New York, 1969. MR 0260724

[GP87] Cornelius Greither and Bodo Pareigis, Hopf Galois theory for separable field extensions, J. Algebra 106 (1987), no. 1, 239-258. MR 878476

[GV17] L. Guarnieri and L. Vendramin, Skew braces and the Yang-Baxter equation, Math. Comp. 86 (2017), no. 307, 2519-2534. MR 3647970

[Hö195] Otto Hölder, Die Gruppen mit quatratfreier Ordnungszahl, Nachr. Königl. Ges. Wiss. Göttingen Math. Phys. 1 (1895), 211-229.

[Koh98] Timothy Kohl, Classification of the Hopf Galois structures on prime power radical extensions, J. Algebra 207 (1998), no. 2, 525-546. MR 1644203

[Koh13] , Regular permutation groups of order mp and Hopf Galois structures, Algebra Number Theory 7 (2013), no. 9, 2203-2240. MR 3152012

[Koh16] _ Hopf-Galois structures arising from groups with unique subgroup of order p, Algebra Number Theory 10 (2016), no. 1, 37-59. MR 3463035

[MM84] M. Ram Murty and V. Kumar Murty, On groups of squarefree order, Math. Ann. 267 (1984), no. 3, 299-309. MR 738255

[NZ19] Kayvan Nejabati Zenouz, Skew braces and Hopf-Galois structures of Heisenberg type, J. Algebra 524 (2019), 187-225. MR 3905210

[Rum07] Wolfgang Rump, Braces, radical rings, and the quantum Yang-Baxter equation, J. Algebra 307 (2007), no. 1, 153-170. MR 2278047

[SV18] Agata Smoktunowicz and Leandro Vendramin, On skew braces (with an appendix by N. Byott and L. Vendramin), J. Comb. Algebra 2 (2018), no. 1, 47-86. MR 3763907

(A. Alabdali) Department of Mathematics, College of Education for Pure Science, University of Mosul, Mosul, Iraq.

Email address: ali.alabdali@uomosul.edu.iq

(N. Byott) Department of Mathematics, College of Engineering, Mathematics and Physical Sciences, University of Exeter, Exeter EX4 4QF U.K.

Email address: N.P.Byott@exeter.ac.uk 